\title{
Flow-cytometric microglial sorting coupled with quantitative proteomics identifies moesin as a highly-abundant microglial protein with relevance to Alzheimer's disease
}

Sruti Rayaprolu', Tianwen Gao ${ }^{1,2}$, Hailian Xiao', Supriya Ramesha', Laura D. Weinstock³, Jheel Shah', Duc M. Duong ${ }^{1,4}$, Eric B. Dammer ${ }^{5}$, James A. Webster $\mathrm{Jr}^{1}$, James J. Lah ${ }^{1}$, Levi B. Wood ${ }^{3}$, Ranjita Betarbet ${ }^{1}$, Allan I. Levey ${ }^{1}$, Nicholas T. Seyfried ${ }^{1,4^{*}}$ and Srikant Rangaraju ${ }^{1 *}$ (D)

\begin{abstract}
Background: Proteomic characterization of microglia provides the most proximate assessment of functionally relevant molecular mechanisms of neuroinflammation. However, microglial proteomics studies have been limited by low cellular yield and contamination by non-microglial proteins using existing enrichment strategies.

Methods: We coupled magnetic-activated cell sorting (MACS) and fluorescence activated cell sorting (FACS) of microglia with tandem mass tag-mass spectrometry (TMT-MS) to obtain a highly-pure microglial proteome and identified a core set of highly-abundant microglial proteins in adult mouse brain. We interrogated existing human proteomic data for Alzheimer's disease (AD) relevance of highly-abundant microglial proteins and performed immuno-histochemical and in-vitro validation studies.

*Correspondence: nseyfri@emory.edu; srikant.rangaraju@emory.edu 'Department of Neurology, Emory University School of Medicine, Whitehead Biomedical Research Building, 615 Michael Street, Atlanta, GA 30322, USA Full list of author information is available at the end of the article

C C The Author(s). 2020 Open Access This article is licensed under a Creative Commons Attribution 4.0 International License, which permits use, sharing, adaptation, distribution and reproduction in any medium or format, as long as you give appropriate credit to the original author(s) and the source, provide a link to the Creative Commons licence, and indicate if changes were made. The images or other third party material in this article are included in the article's Creative Commons licence, unless indicated otherwise in a credit line to the material. If material is not included in the article's Creative Commons licence and your intended use is not permitted by statutory regulation or exceeds the permitted use, you will need to obtain permission directly from the copyright holder. To view a copy of this licence, visit http://creativecommons.org/licenses/by/4.0/ The Creative Commons Public Domain Dedication waiver (http://creativecommons.org/publicdomain/zero/1.0/) applies to the data made available in this article, unless otherwise stated in a credit line to the data. 
(Continued from previous page)

Results: Quantitative multiplexed proteomics by TMT-MS of CD11b + MACS-enriched ( $N=5$ mice) and FACSisolated ( $N=5$ mice), from adult wild-type mice, identified 1791 proteins. A total of 203 proteins were highly abundant in both datasets, representing a core-set of highly abundant microglial proteins. In addition, we found 953 differentially enriched proteins comparing MACS and FACS-based approaches, indicating significant differences between both strategies. The FACS-isolated microglia proteome was enriched with cytosolic, endoplasmic reticulum, and ribosomal proteins involved in protein metabolism and immune system functions, as well as an abundance of canonical microglial proteins. Conversely, the MACS-enriched microglia proteome was enriched with mitochondrial and synaptic proteins and higher abundance of neuronal, oligodendrocytic and astrocytic proteins. From the 203 consensus microglial proteins with high abundance in both datasets, we confirmed microglial expression of moesin (Msn) in wild-type and 5xFAD mouse brains as well as in human AD brains. Msn expression is nearly exclusively found in microglia that surround $A \beta$ plaques in $5 x F A D$ brains. In in-vitro primary microglial studies, Msn silencing by siRNA decreased A $\beta$ phagocytosis and increased lipopolysaccharide-induced production of the pro-inflammatory cytokine, tumor necrosis factor (TNF). In network analysis of human brain proteomic data, Msn was a hub protein of an inflammatory co-expression module positively associated with AD neuropathological features and cognitive dysfunction.

Conclusions: Using FACS coupled with TMT-MS as the method of choice for microglial proteomics, we define a core set of highly-abundant adult microglial proteins. Among these, we validate Msn as highly-abundant in plaqueassociated microglia with relevance to human AD.

Keywords: Microglia, Proteomics, Mass spectrometry, FACS, MACS, Alzheimer's disease

\section{Background}

Microglia are the resident macrophages and primary immune effectors in the brain that are central to neuroinflammatory mechanisms of Alzheimer's disease (AD) pathogenesis $[1,2]$. The causal role of microglia in $\mathrm{AD}$ is evidenced by data from recent unbiased genome-wide association studies (GWAS) that have identified several single-nucleotide polymorphisms (SNPs) in immune related genes as independent risk factors for late-onset $\mathrm{AD}$ $[3,4]$. Approximately two thirds of the 29 susceptibility genes are exclusively or most highly expressed in microglia $[3,4]$. In human $\mathrm{AD}$ and mouse models of $\mathrm{AD}$ pathology brains, microglia with activated morphology are frequently found surrounding amyloid-beta $(A \beta)$ plaques $[5,6]$. Microglial depletion in mouse models of AD pathology also results in decreased neuropathological features of $\mathrm{AD}[7,8]$. Overall, these convergent findings implicate microglia in the pathogenesis of $\mathrm{AD}$, but whether microglia are protective $[5,6]$ or detrimental $[1$, 2] remains elusive.

Defining the molecular characteristics of microglia in the brain has been primarily undertaken using transcriptomic strategies. Microglial isolation required for these studies can be performed by fluorescence activated cell sorting (FACS), magnetic activated cell sorting (MACS), differential gradient centrifugation, and immunopanning, resulting in collection of intact microglia from fresh mouse and human brain tissues [9-12]. Next generation RNA-sequencing has enabled the quantification of transcripts in low numbers of microglia isolated from brain tissue. Transcriptomic profiles of isolated microglia from animal models have revealed regional heterogeneity of microglia and have shown that progressive neuropathology in AD models results in a shift from homeostatic to transcriptionally distinct disease-related phenotypes [1318]. Though comprehensive transcriptomic analyses have proven useful in the characterization of microglia, there is still a gap in our understanding of what transcriptional profiles mean biologically and how they relate to protein function. For instance, mRNA and protein expression profiles differ quantitatively, temporally, and spatially [19]. Several studies have shown that transcriptlevel and protein-level expression data are discordant because of post-transcriptional processes such as mRNA regulation, post-translational protein modifications, and protein recycling and degradation [20, 21]. ProteinmRNA correlation coefficients reach no higher than 0.47 in complex tissues like the brain and this relationship is poorly understood in acutely isolated brain cell types, including microglia [22]. Therefore, global and comprehensive proteomic profiling of isolated microglia can make the most proximate assessment of their biological functions, rather than transcriptional profiling.

Proteomic studies of microglia have primarily used invitro cultured or neonatal microglia [22-24] and few studies have used $\mathrm{CD}_{11 \mathrm{~b}^{+}}$MACS enrichment to obtain $>90 \%$ microglia for downstream proteomic analyses. In an effort to elucidate proteomes of adult microglia, we have recently successfully performed quantitative tandem mass tag (TMT) mass spectrometry (MS) of acutely isolated $\mathrm{CD}_{11 \mathrm{~b}^{+}}$MACS-enriched microglia from adult (6-7mo) mice of normal, acute neuroinflammatory 
(lipopolysaccharide [LPS]-treatment), and chronic neurodegenerative (5xFAD model of $\mathrm{AD}$ ) states [25]. This deep and comprehensive proteomic study of isolated mouse microglia identified over 4000 proteins and allowed us to identify unique microglial proteomic changes in mouse models of AD pathology. This study has revealed novel roles for microglial proteins in human neurodegeneration and emphasizes the value of applying state-of-the-art proteomics methods to resolve cell-type specific contributions to disease [25]. Despite microglial enrichment, these data also suggested that MACS still suffers from contamination by proteins from other cell types, most likely due to non-cellular debris that remain in MACS microglial preparations. In support of this limitation, we found that despite $>90 \%$ microglial cell enrichment by MACS, some of the proteins with highest abundance included oligodendrocytic (Mbp) and astrocytic (Gfap) proteins. Therefore, optimization of microglia purification strategies that are best suited for microglial proteomic studies are warranted prior to comprehensive characterization in disease models as well as in human tissues.

While both MACS-enriched and FACS-based cell purification yield high cell purity, protein-rich noncellular elements can be definitively excluded by FACS and not by MACS. In this study, we compared the proteomes of MACS-enriched and FACS-isolated CD11b ${ }^{+}$ microglia from adult mouse brain to identify a consensus set of highly-abundant microglial proteins and characterize and contrast the purification efficiencies of each technique. Based on the observed enrichment of microglial proteins and depletion of neuronal, astrocytic, and oligodendroglial proteins, we show that the FACSisolation approach is the preferred approach for microglial proteomic studies. Using the consensus list of highly-abundant microglial proteins, we validated microglial specific expression of two proteins, namely Msn and Cotl1, in mouse and human brain. We performed in-vitro functional studies confirming a role for Msn in $A \beta$ phagocytosis and pro-inflammatory stimulusinduced cytokine release. Importantly, we found Msn as a key member of a glial and inflammatory group of coexpressed proteins that is strongly associated with $\mathrm{AD}$ neuropathologies and cognitive dysfunction in human AD cases.

\section{Methods}

\section{Animals}

Mice were housed in the Department of Animal Resources at Emory University under a 12-h light/12-h dark cycle with ad libitum access to food and water. All procedures were approved by the Institutional Animal Care and Use Committee of Emory University and were in strict accordance with the National Institute of
Health's "Guide for the Care and Use of Laboratory Animals."

\section{Acute isolation of CD11b-positive microglia}

The isolation workflow is shown in Fig. 1a. Male 4month-old $\mathrm{C} 57 \mathrm{Bl} / 6 \mathrm{~J}$ mice $(N=10)$ were anesthetized with isoflurane, followed by exsanguination and cardiac perfusion with $30 \mathrm{~mL}$ of ice-cold $1 \times$ phosphate buffered saline $(1 \times$ PBS $)$. The brain was immediately dissected and mechanically dissociated over a $40 \mu \mathrm{m}$ cell strainer. Subsequently, the suspension was centrifuged for $5 \mathrm{~min}$ at $800 \times g$ at room temperature (RT), supernatant was carefully decanted, and pellet was resuspended in $6 \mathrm{~mL}$ of $35 \%$ stock isotonic Percoll (SIP) solution diluted with $1 \times$ HBSS (SIP: 9 parts 100\% Percoll and 1 part $10 \times$ HBSS). The cell suspension was transferred to a new 15 $\mathrm{mL}$ conical and $3 \mathrm{~mL}$ of $70 \%$ SIP was slowly underlaid. The established gradient was centrifuged for $25 \mathrm{~min}$ at $800 \times g$ with no brake at $15^{\circ} \mathrm{C}$. The top floating myelin layer was aspirated and $3 \mathrm{~mL}$ from the $35-70 \%$ interphase, containing the mononuclear cells (Fig. 1a), was collected without disturbing the $70 \%$ layer. The mononuclear cells were washed with $6 \mathrm{~mL}$ of $1 \times \mathrm{PBS}$, centrifuged for $5 \mathrm{~min}$ at $800 \times g$, and cell pellet was resuspended in $100 \mu \mathrm{l}$ of $1 \times$ PBS.

Isolated mononuclear cells from five brains were then further purified by $\mathrm{CD} 11 \mathrm{~b}$ positive selection using MACS columns ( $N=5$ mice, $>90 \%$ enrichment of $\mathrm{CD}_{11 b^{+}}$microglia [25]) (Miltenyi Biotec, Cat. No. 130093-636) to selectively enrich microglia and brain mononuclear phagocytes (Fig. 1b). Cells were pelleted, resuspended in $200 \mu \mathrm{L}$ of lysis buffer ( $8 \mathrm{M}$ urea, $100 \mathrm{mM}$ $\mathrm{NaHPO}_{4}, \mathrm{pH} 8.5$ ) with HALT protease and phosphatase inhibitor cocktail (ThermoFisher, Cat. No. 78446), and frozen at $-80^{\circ} \mathrm{C}$ until protein digestion. Mononuclear cells from the remaining five brains were labeled with APC-Cy7 rat anti-CD11b antibody (BD Pharmingen, Cat. No. 557657) for $30 \mathrm{~min}$ at RT, washed, and kept on ice until sorting. Compensation experiments were performed using OneComp beads (Thermofisher, Cat. No. 01-1111-42). Live mononuclear cells were gated based on FSC-A/SSC-A, then gated based on FSC-A/FSC-H to identify single cells (Fig. 1b), as previously described [26, 27]. The cells were further gated for APC-Cy7 fluorescence, sorted using a BD FACSAria II cell sorter directly into $1.5 \mathrm{~mL}$ tubes containing $200 \mu \mathrm{L}$ of lysis buffer containing protease and phosphatase inhibitors, and frozen at $-80{ }^{\circ} \mathrm{C}$ until protein digestion. Negative controls included unstained mononuclear cells.

In order to allow for a fair comparison between both approaches, we intentionally sorted $\mathrm{CD}_{11} \mathrm{~b}^{+}$myeloid cells (predominantly microglia) rather than using a $\mathrm{CD} 11 \mathrm{~b}^{+} \mathrm{CD} 45^{\mathrm{int}}$ selection strategy. Since live/dead gating is not feasible for MACS-enrichment, we intentionally 


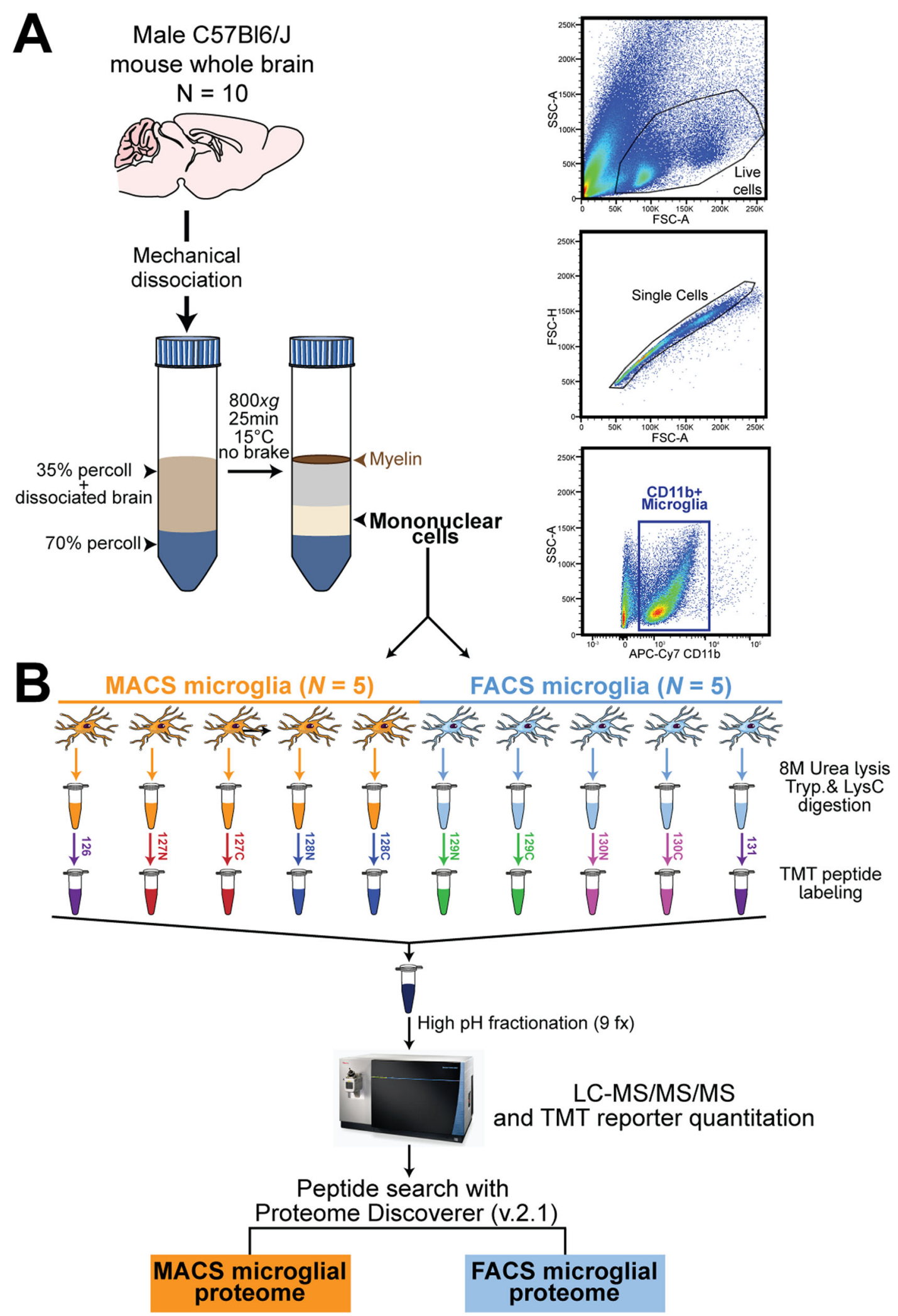

Fig. 1 (See legend on next page.) 
(See figure on previous page.)

Fig. 1 Study design and analytic approach for comprehensive quantitative proteomic analysis of isolated mouse microglia. a Workflow summarizing isolation and purification of $\mathrm{CD}_{11} \mathrm{~b}^{+}$brain immune cells from 4-month-old male C57B/6/J wild-type mice $(\mathrm{N}=10)$. Following mechanical dissociation of fresh, whole mouse brains and percoll density centrifugation, mononuclear cells were enriched for $\mathrm{CD}_{11} \mathrm{~b}^{+}$microglia cells by magnetic activating cell sorting (MACS, $N=5$ mice) or isolated via fluorescent activated cell sorting (FACS, $N=5$ mice). Representative flow cytometry gating strategy and antibody separation using CD11b-APC/Cy7 antibody for isolation of microglia. b Proteomic workflow for tandem mass tag (TMT) mass spectrometry (MS) based quantification. All 10 microglia samples were lysed in $8 \mathrm{M}$ urea, digested with LysC and Trypsin, and peptides were labeled using one 10-plex TMT kit. A total of 5 individual MACS-enriched microglia samples were dedicated to the first five channels $(126,127 \mathrm{~N}, 127 \mathrm{C}, 128 \mathrm{~N}, 128 \mathrm{C})$ and 5 individual FACS-isolated microglia samples were dedicated to the last five channels (129 $\mathrm{N}, 129 \mathrm{C}, 130 \mathrm{~N}, 130 \mathrm{C}, 131)$. After labeling, the samples were combined and fractionated by off-line high pH fractionation ( $n=9$ fractions [fx]). Each fraction was analyzed and quantified by synchronous precursor selection (SPS)-MS3 on an Orbitrap Fusion mass spectrometer. Peptide search on raw files were conducted with Proteome Discoverer (V2.1) in order to obtain the MACS microglia proteome and FACS microglia proteome

did not use live/dead exclusion in the gating strategy during FACS. However, we confirmed in independent experiments that cell viability using our FACS strategy is

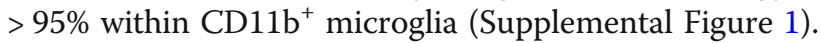
We have also previously shown that CD $45^{\text {high }}$ braininfiltrating macrophages represent $<5 \%$ of cells within

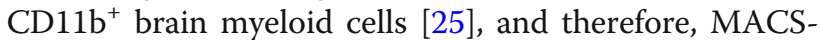

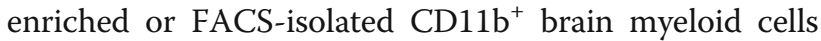
are referred to as microglia in this manuscript.

\section{Protein digestion and TMT labeling}

Isolated microglia in lysis buffer were sonicated for $3 \mathrm{cy}-$ cles consisting of $5 \mathrm{~s}$ of active sonication at $30 \%$ amplitude followed by $15 \mathrm{~s}$ on ice. Protein concentration was determined by bicinchoninic acid (BCA) assay (Pierce, Cat. No. 23225). Protein digestion was performed as previously described [28]. Briefly, $6 \mu \mathrm{g}$ of protein for each sample was reduced with $1 \mathrm{mM}$ dithiothreitol (DTT) at room temperature for $30 \mathrm{~min}$ and alkylated by $5 \mathrm{mM}$ iodoacetamide (IAA) in the dark for $30 \mathrm{~min}$. Samples were then diluted (8-fold) with $50 \mathrm{mM}$ triethylammonium bicarbonate (TEAB), digested overnight with Lysyl endopeptidase (Wako, Cat. No. 127-06621) at 1:100 (w/ $\mathrm{w})$. The peptide solutions were acidified to a final concentration of $1 \%$ formic acid (FA) and $0.1 \%$ triflouroacetic acid (TFA), desalted with a $\mathrm{C}_{18}$ Sep-Pak column (Waters, Cat. No. WAT054945) and dried down in a vacuum centrifuge (SpeedVac Vacuum Concentrator). TMT labeling of peptides was performed according to manufacturer's instructions and as previously described [28]. One batch of 10-plex TMT kit (Thermo Fisher, Cat. No. 90110) was used to label all 10 samples (Fig. 1b). All 10 channels were then combined and dried in a vacuum centrifuge.

\section{High $\mathrm{pH}$ reversed-phase peptide fractionation}

High $\mathrm{pH}$ reversed-phase peptide fractionation kit was used to perform desalting and fractionation as per manufacturer's protocol (ThermoFisher, Cat. No. 84868). Briefly, the dried sample containing all 10 TMT channels was reconstituted with $300 \mu \mathrm{L}$ of $0.1 \%$ TFA and added to conditioned reversed-phase fractionation spin columns containing $20 \mathrm{mg}$ of resin in a 1:1 water/DMSO slurry. A sequential gradient of increasing acetonitrile $(\mathrm{ACN})$ concentrations in a high-pH elution solution ( $0.1 \%$ triethylamine) was applied to the columns to elute bound peptides into 9 different fractions collected by centrifugation at $3000 \times g$. Each fraction was dried in a vacuum centrifuge and stored at 4 degrees until mass spectrometry.

\section{Mass spectrometry analysis and TMT data acquisition}

Assuming equal distribution of peptide concentration across all 9 fractions, $10 \mu \mathrm{L}$ of loading buffer (0.1\% TFA) was added to each fraction and $2 \mu \mathrm{L}$ was separated on a $25 \mathrm{~cm}$ long by $75 \mu \mathrm{m}$ internal diameter fused silica column (New Objective, Woburn, MA) packed in-house with $1.9 \mu \mathrm{m}$ Reprosil-Pur C18-AQ resin. The LC-MS/ MS platform consisted of a Dionex RSLCnano UPLC coupled to an Orbitrap Fusion mass spectrometer with a flex nano-electrospray ion source (ThermoFisher). Sample elution was performed over a 120 min gradient with a constant flow rate of $300 \mathrm{nl} / \mathrm{min}$. The gradient consisted of multiple steps starting at $3 \%$ and going to $7 \%$ Buffer B (0.1\% formic acid in ACN) over 5 mins, from 7 to $30 \%$ Buffer B over 80 mins, from 30 to $60 \%$ Buffer B over 5 mins, from 60 to $99 \%$ Buffer B over 2 mins, constant at $99 \%$ for 8 mins and immediately back to $1 \%$ Buffer $\mathrm{B}$ for 20 mins. The mass spectrometer was operated in positive ion mode and utilized the synchronous precursor selection (SPS)-MS3 method for reporter ion quantitation as described [28] with a top speed cycle time of $3 \mathrm{~s}$. The full scan range was $400-1500 \mathrm{~m} / \mathrm{z}$ at a nominal resolution of 120,000 at $200 \mathrm{~m} / \mathrm{z}$ and automatic gain control (AGC) set to $4 \times 10^{5}$. Tandem MS/MS Collision-induced dissociation (CID) spectra were collected in the ion trap with normalized collision energy set to $35 \%$, max injection time set to $35 \mathrm{~ms}$ and AGC set to $1 \times 10^{4}$. Higher energy collision dissociation (HCD) SPS-MS3 of the top 10 product ions at $65 \%$ normalized collision energy (CE) were collected in the orbitrap with a resolution of 60,000, a max injection time of $100 \mathrm{~ms}$ and an AGC setting of $5 \times 10^{4}$. 


\section{Protein identification and quantification}

Raw files from Orbitrap Fusion were processed using Proteome Discoverer (v2.1) and MS/MS spectra were searched against UniProt Mouse proteome database (54, 489 total sequences) as previously reported [25]. SEQUEST parameters were specified as: trypsin enzyme, two missed cleavages allowed, minimum peptide length of 6, TMT tags on lysine residues and peptide $\mathrm{N}$-termini $(+229.162932 \mathrm{Da})$ and carbamidomethylation of cysteine residues $(+57.02146 \mathrm{Da})$ as fixed modifications, oxidation of methionine residues $(+15.99492 \mathrm{Da})$, and deamidation of asparagine and glutamine $(+0.984 \mathrm{Da})$ as a variable modification, precursor mass tolerance of 20 ppm, and a fragment mass tolerance of 0.6 Da. Peptide spectral match (PSM) error rates were determined using the target-decoy strategy coupled to Percolator [29] modeling of true and false matches. Reporter ions were quantified from MS3 scans using an integration tolerance of $20 \mathrm{ppm}$ with the most confident centroid setting. An MS2 spectral assignment false discovery rate (FDR) of less than $1 \%$ was achieved by applying the targetdecoy strategy. Following spectral assignment, peptides were assembled into proteins and were further filtered based on the combined probabilities of their constituent peptides to a final FDR of $1 \%$. In cases of redundancy, shared peptides were assigned to the protein sequence with the most matching peptides, thus adhering to principles of parsimony. The search results and TMT quantification as well as raw LC-MS/MS files are included in the ProteomeXchange online repository with identifier PXD015652. We included proteins with TMT abundance values in at least 3 of the 5 replicates per group, as well as proteins present within all 5 replicates in one group and absent in the other group (present only in MACS: total $n=4$ ), but also display a high protein FDR confidence ( $n=2$, Gpm6b \& Sh3gl2). Additionally, even though TMT labeling limits missing values, normalized abundances of zero were imputed as the lowest TMT abundance value in the dataset.

\section{Differential expression analysis}

Differentially enriched or depleted proteins (unadjusted $p \leq 0.05)$ were identified by unpaired $\mathrm{t}$-test comparing the five FACS-isolated microglia samples and the five MACS-enriched microglia samples. Differential expression (FACS/MACS) is presented as volcano plots which display all proteins that either arise from expression of one of the proteins or gene products in the cell typespecific enrichment lists [11, 22]. Significance of differentially expressed protein overlap with cell type-specific enriched protein marker lists was assessed using a one tailed Fisher's exact test and corrected for multiple comparisons by the FDR (Benjamini-Hochberg) method (Supplemental Table 1).

\section{Gene ontology enrichment analysis}

Gene Ontology (GO) functional annotation of differentially expressed proteins was performed using GO-Elite v1.2.5 as previously published [30,31], with a minimum of five genes per ontology, meeting Fisher exact significance of $p<0.05$, i.e., a Z-score greater than 1.96 . The background gene list for GO-Elite consisted of unique gene symbols for all proteins identified and quantified in our dataset $(n=1791)$. Input lists included proteins that were significantly differentially enriched or depleted $(p<$ 0.05 by unpaired $t$-test, unadjusted) and with $a \geq 2$-foldchange in abundance comparing FACS-isolated with MACS-enriched microglial proteomes (Supplemental Table 2).

\section{Cell-type enrichment analysis}

Cell-type enrichment was performed by crossreferencing the number of differentially enriched proteins in our FACS vs. MACS microglial proteomes with cell type-specific gene lists from mass-spectrometry based proteomics [22] (termed "reference cell-type proteome") and RNA-Seq [11] (termed "reference celltype transcriptome") of the following isolated mouse brain cell types: microglia, oligodendrocytes, astrocytes, neurons, and endothelial cells. Methods for determining cell-type enrichment of each protein or gene have been previously published and used in prior proteomic analyses [30, 32]. Of note, purified mouse endothelial proteomes have yet to be published.

\section{Immunofluorescence staining}

Brains were isolated from wild-type $\mathrm{Cx} 3 \mathrm{cr} 1^{\mathrm{CreER}-\mathrm{YFP}}$ mice $(N=4$; Jackson Stock No. 021160) and Cx3cr1 ${ }^{\text {CreER-YFP }}$ mice crossed with $5 x$ FAD mice $(N=6)$ at 6-7 months of age. Cx3cr1 $1^{\text {CreER-YFP }}$ mice constitutively express YFP in microglia [33, 34]. Brains sections from 9 to 10 month old wild-type (C57Bl6/J, $N=3)$ and $5 x \mathrm{xAD}(N=4)$ were used for CD31/Msn and GFAP/ Msn co-immunostaining. Briefly, the mice were anesthetized with isoflurane and perfused transcardially with 30 $\mathrm{mL}$ of $1 \times$ PBS. The brains were immediately removed and hemisected along the sagittal midline. The left hemisphere was immersion-fixed in $4 \%$ paraformaldehyde for $24 \mathrm{~h}$, washed 3 times with $1 \times \mathrm{PBS}$, and transferred to $30 \%$ sucrose for another $24 \mathrm{~h}$. Subsequently, the brains were cut into $30 \mu \mathrm{m}$ thick sagittal sections using a cryostat. For immunofluorescence staining, 3-4 brain sections from each mouse were thoroughly washed to remove cryopreservative, blocked in $8 \%$ normal horse serum diluted in $1 \times$ tris buffered saline (TBS) and $0.1 \%$ Triton-X for $1 \mathrm{~h}$, and incubated with primary antibodies diluted in $1 \times$ PBS overnight (1:200 goat anti-GFP [Rockland, Cat. No. 600-101-215], 1:100 rabbit anti-Msn [Abcam, Cat. No. ab52490], 1:25 rat anti-CD31 [BD 
Bioscience, Cat. No. 550274]. 1:500 mouse anti-GFAP [Thermo, Cat. No. 14-9892-82], 1:100 rabbit anti-Cotl1 [Sigma-Aldrich, Cat. No. HPA008918]). For A $\beta$ staining, $5 x F A D$ brain sections were treated with $80 \%$ formalin for $3 \mathrm{~min}$, washed with for $10 \mathrm{~min}$ per wash, blocked, and incubated overnight with mouse anti- $\beta$-amyloid 4G8 clone (1:500, [BioLegend, Cat. No. 800701]). Following washes and incubation in the appropriate fluorophoreconjugated secondary antibody (1:500, anti-goat FITC, anti-rat Alexa 488, anti-mouse FITC, anti-mouse DyLight 405, anti-rabbit Rhodamine-red) for $1 \mathrm{~h}$, sections were mounted on slides with mounting media containing DAPI (Sigma-Aldrich, Cat. No. F6057) for nuclear staining. Representative images of the same region in the cortex were taken using the Leica SP8 multiphoton confocal microscope and all images processing was performed using Fiji software [35].

\section{Quantitative analysis of msn immunofluorescence}

Confocal micrographs (40x magnification) from agematched WT $(N=3)$ and $5 x F A D(N=3)$ brains were used for quantitative analysis of Msn protein expression in microglia. We sampled ramified microglia from WT mice and plaque-associated microglia from 5xFAD mice. Regions of interest containing a single microglial cell were drawn and Msn immunoreactivity per microglial cell was measured by sampling 4-7 microglia per section from each mouse. Background subtraction was performed before analysis. Average Msn immunoreactivity (intensity normalized to area of region of interest) in WT and 5xFAD microglia was statistically compared using unpaired t-test. Image analysis was performed using ImageJ.

\section{Primary mouse microglia isolation}

Primary mouse microglial cultures were established using published isolation and enrichment protocols [36, 37]. Briefly, brains from wild-type C57Bl6/J mice (age P0-3) were removed immediately after euthanization and digested with Trypsin (ThermoFisher, Cat. No. 25300054) for $15 \mathrm{~min}$ at $37^{\circ} \mathrm{C}$. Subsequently, Trypsin reaction was quenched with $20 \mathrm{~mL}$ of DMEM (Dulbecco's Modified Eagle Medium, ThermoFisher, Cat. No. 10569-010) with 10\% fetal bovine serum (FBS) and 1\% penicillin streptomycin glutamine (ThermoFisher, and Cat. No. 10378016). The floating myelin debris was removed, and the remaining cell suspension was filtered through a $40 \mu \mathrm{m}$ cell strainer and centrifuged at $800 \times g$ for $5 \mathrm{~min}$. The cell pellet was washed with DMEM/10\% FBS followed by CD11b positive selection (Miltenyi Biotec, Cat. No. 130-093-636) using mini-MACS (Miltenyi Biotec, Cat. No. 130-042-201) columns. The CD11b positive microglia were seeded in poly-L-lysine-coated wells and cultured in DMEM at $37^{\circ} \mathrm{C}, 5 \% \mathrm{CO}_{2}$. The medium was replaced with fresh medium after $24 \mathrm{~h}$ (Fig. 4a).

\section{Small interfering RNA (siRNA) transfection studies}

Primary microglia were transfected with $40 \mathrm{nM}$ (final concentration) of either Msn siRNA (Santa Cruz Biotechnology, Cat. No. sc-35,956) or nonspecific sham siRNA (Santa Cruz Biotechnology, Cat. No. sc-37,007) using Lipofectamine $^{\mathrm{TM}}$ RNAiMAX (Invitrogen, Cat. No. 13778100) and Opti-MEM (Invitrogen, Cat. No. 31985-062). After $48 \mathrm{~h}$, the efficiency of siRNA-mediated gene silencing was confirmed by quantitative reverse-transcriptase polymerase chain reaction (qRT-PCR) (Fig. 4b).

\section{Quantitative reverse transcriptase PCR (qRT-PCR)}

Total RNA from microglia was extracted using Trizol (Invitrogen, Cat. No. 15596026) and an RNeasy mini extraction kit (Qiagen, Cat. No. 74104) according to the manufacturer's instructions. RNA concentration was assessed using Nanodrop and cDNA was synthesized using high-capacity cDNA reverse transcription kit (Applied Biosystems, Cat. No. 4368814). Quantitative real-time PCR was performed on a 7500 Fast Real-time PCR System (Applied Biosystems) using cDNA, TaqMan PCR Master Mix (Applied Biosystems, Cat. No. 4304437), and gene-specific TaqMan probes (Applied Biosystems) against Moesin (Mm00447889_m1) and Gapdh (Mm99999915_g1). Each primer set was run in triplicate for each RNA sample. Gene expression was normalized to the internal housekeeping gene GAPDH in primary microglial cells, and relative expression was calculated for each gene using the $2 \Delta \Delta \mathrm{CT}$ method after normalizing to the control sample (sham siRNA) [38].

\section{Primary microglia activation by lipopolysaccharide}

Microglia were activated with $2 \mathrm{ng} / \mathrm{mL}$ of lipopolysaccharide (LPS; Sigma Aldrich, Cat. No. L4391, Escherichia coli 0111:B4) after $24 \mathrm{~h}$ of siRNA exposure. Cells were collected after $24 \mathrm{~h}$ of LPS treatment for phagocytosis assays and the supernatants were collected for cytokine assays (Fig. 4a).

\section{Fluorescent fibrillar $A \beta 42$ phagocytosis flow cytometric assay}

After in-vitro exposure to sham or Msn siRNA and/or LPS stimulus, primary microglia were treated with $2 \mu \mathrm{M}$ (final concentration) of fibrillar fluorescent $\mathrm{A} \beta 42$ conjugated to HiLyte Fluor 488 (fA $\beta 42-488$, AnaSpec, Cat. No. AS-60479-01) for $1 \mathrm{~h}$ at $37^{\circ} \mathrm{C}$. fA $\beta 42-488$ was prepared by combining $100 \mu \mathrm{g}$ of peptide with $20 \mu \mathrm{L}$ of $1 \%$ ammonium hydroxide $\left(\mathrm{NH}_{4} \mathrm{OH}\right)$ and immediately diluted to a final concentration of $100 \mu \mathrm{M}$ with $1 \times$ PBS. After incubation at room temperature for 6 days, fA $\beta 42-488$ was used for the phagocytosis assay [26, 27]. 
Subsequent to incubation, cells were treated with trypsin, washed with $1 \times \mathrm{PBS}$, and labeled with CD45-PECy7 antibody (BD Biosciences, Cat. No. 552848). Compensation experiments and gating were performed as described above and previously [26, 27]. Single live cells were gated based on CD45-PE-Cy7 fluorescence and fA $\beta 42-488$ positivity to determine the phagocytic uptake of $\mathrm{A} \beta 42$ within live $\mathrm{CD} 45^{+}$microglia (expressed as a proportion of microglia that were positive for $A \beta 42$ fluorescence). Negative controls included microglia incubated with antibodies except for fAß42-488. We have previously demonstrated loss of $\mathrm{fA} \beta 42-488$ uptake by microglia after cytochalasin D treatment, confirming that this assay measures actin-dependent phagocytic processes $[26,27]$.

\section{Cytokine and chemokine multiplex assay}

After in-vitro exposure to siRNA and/or LPS stimulus, primary microglia culture supernatants were collected, centrifuged to remove debris, aliquoted, and stored at $80^{\circ} \mathrm{C}$. For cytokine analyses, one aliquot of culture supernatant was diluted to $24 \%$ in Milliplex Assay Buffer and analyzed using a Milliplex MAP Mouse Cytokine/ Chemokine Multiplex Assay (Millipore Sigma, Cat No. MCYTOMAG-70 K) per manufacturer's instructions for the following custom collection of analytes: GM-CSF, IFN- $\gamma$, IL-10, IL-1 $\alpha$, IL-2, IL-4, IL-6, IP-10/CXCL10, MCP-1/CCL2, MIP-1 $\alpha / C C L 3$, TNF- $\alpha$, M-CSF, VEGF-A, G-CSF, RANTES [39]. Fluorescent intensity readings for each cytokine were compared across experimental groups and performed in biological triplicates (Fig. 4a).

\section{Existing transcriptomic and proteomic datasets used for comparative analyses \\ Human brain proteomic data}

Dataset 1 Msn protein abundance, neuropathological, and cognitive data were obtained from a recently published study [40] (Synapse Web Portal https://doi.org/10. 7303/syn20933797) and are shown in Fig. 5b and d. In this study, dorsolateral prefrontal cortex (DLPFC) tissues from non-disease control $(N=91)$, asymptomatic $\mathrm{AD}$ (AsymAD, $N=98)$, and $\mathrm{AD}(N=230)$ cases were analyzed by mass spectrometry based proteomics using label-free quantitation (LFQ). A total of 3334 proteins were quantified with fewer than $50 \%$ missing values across all cases and were used to make a protein coexpression network using the weighted correlation network analysis (WGCNA) algorithm. This dataset was selected because it is currently the largest quantitative mass spectrometry-based proteomics analysis that generated a consensus AD brain protein co-expression network across multiple study centers
Dataset 2 Msn protein abundance values were obtained from a recently published study [40] (Synapse Web Portal https://doi.org/10.7303/syn20933797) and are shown in Supplemental Figure 6A. In this study, control $(N=$ $43), \mathrm{AD}(N=47)$, f rontotemporal dementia with TDP43 pathology (FTLD-TDP, $N=29$ ), amyotrophic lateral sclerosis (ALS, $N=54$ ), and Parkinson's disease and Parkinson's disease dementia (PD/PDD, $N=76$ ) DLPFC brain tissues were analyzed by LFQ-MS and 2919 proteins were identified. This dataset was selected to determine whether increased Msn levels in AD brain replicated in a different cohort and to see if this increase is selective to $\mathrm{AD}$

Dataset 3 Msn protein abundance values were obtained from a recently published study (Synapse Web Portal https://doi.org/10.7303/syn20933797) [40] and are shown in Supplemental Figure 6B. Control $(N=13)$, AsymAD $(N=13)$, and $\operatorname{AD}(N=22)$ brain tissue from precuneus were analyzed using LFQ-MS and 3348 proteins were identified. This dataset was selected to assess whether Msn levels increase in another brain region in addition to the DLPFC from Dataset 1 and because the precuneus is a region affected early in $\mathrm{AD}$ pathogenesis

Dataset 4 Msn protein abundance data were obtained from a recently published study by Drummond et al. [41] and are shown in Supplemental Figure 6C. In this study, LFQ-MS was performed on $\mathrm{A} \beta$ plaques microdissected from the hippocampus and adjacent entorhinal cortex of rapidly progressive $\mathrm{AD}(\mathrm{rpAD}, N=22)$ and sporadic $\mathrm{AD}$ (spAD, $N=22$ ) cases. Approximately 900 proteins were quantified from every case. This is the first large-scale proteomic study that analyzed the $A \beta$ plaque proteome and thus, was used to validate our observation of Msn positive microglia surrounding $A \beta$ plaques in the 5xFAD mouse brain

\section{Human brain transcriptomic data}

Msn gene expression in pre-frontal cortex from control $(N=157)$ and $\operatorname{AD}(N=308)$ brains was obtained from Zhang et al. (GEO: GSE44772) [42]. For each sample, 39, 579 transcripts were profiled which represent 25,242 known and 14,337 predicted gene-expression traits. The gene-expression was adjusted for age and sex, postmortem interval (PMI) in hours, and sample pH and RNA integrity number (RIN).

\section{Mouse brain proteomic data}

We obtained Msn abundance from an existing proteomic dataset [43] (PXD006214) of WT, 5xFAD (A $\beta$ pathology), JNPL3 (tau pathology), and 5xFAD crossed with JNPL3 (A $\beta$ and tau pathologies) mouse lines. In this study, quantitative mass spectrometry using TMT tags 
was performed on hippocampi from mice of all four lines at 4 months, 7 months, and 10 months of age with three biological replicates for each line and age. The samples were randomized into four 10-plex TMT batches and a total of 6964 proteins were identified after statistical processing. This dataset was selected because it provides a deep proteome of two mouse models of $\mathrm{AD}$ pathology, $A \beta$ and tau, at various ages.

\section{Mouse brain transcriptomic data}

Msn gene expression in cortical tissue from WT and $5 x F A D$ mice at 3 months, 6 months, and 12 months of age was obtained from Bai et al. [44] (GEO: GSE140286). There were 2-3 animals per genotype and age. The gene-level fragments per kilobase of transcript per million (FPKM) values were computed from the read counts for each gene.

\section{Immunostaining of human brain tissues}

Cryopreserved, pathology-confirmed $\mathrm{AD}(N=10,5$ males, 5 females, average age at death $=72.7$ ) and nondisease control $(N=10,5$ males, 5 females, average age at death $=61.4)$ frontal cortex tissue sections $(50 \mu \mathrm{m}$ thick) were obtained from the Emory Neuropathology/ Histochemistry Core. Sections were immunostained for Msn (1:100, Abcam, Cat. No. ab52490) using DAB as the chromophore. Adjacent sections were processed for double fluorescent immunolabeling for Msn and $A \beta$ beta protein (4G8, 1:1000, Signet, Cat. No. 9220-02). Sections were incubated in primary antibody for $72 \mathrm{~h}$ followed by a $2 \mathrm{~h}$ incubation in secondary antibodies conjugated to appropriate fluorophores. Secondary antibodies used were Alexa 488 goat anti-mouse/rabbit (1:200, Molecular Probes, Eugene, OR) and Cy3 or biotinylated goat antimouse/rabbit (1:200, Jackson Immunoresearch Labs). Subsequently, IF sections were incubated in diamidino2-phenylindole (DAPI) for $10 \mathrm{~min}$ (1:5000, Molecular Inc. Probes, Eugene, OR). For controls primary antibodies were (a) either pre-absorbed with specific peptide sequence or (b) were omitted. Autofluorescence was eliminated (Chemicon, Cat. No. 2160), sections were mounted, and coverslipped. Images were captured using an Olympus bright-field and fluorescence microscope and camera (OlympusBX51). For final output, images were processed using Adobe Photoshop software.

\section{Statistical considerations}

Our statistical approach for MS studies is described above. One-way analysis of variance (ANOVA) was used to identify group-wise differences and post-hoc Tukey's test was used to identify pairwise differences $(p<0.05$ considered significant). All analyses were performed using Graphpad Prism (v7).

\section{Results}

Comparative proteomic analysis of MACS and FACS-based strategies reveals highly-abundant core microglial proteins and highlights differences between both purification strategies

We applied TMT-MS to obtain the proteomes of $\mathrm{CD}_{11 \mathrm{~b}^{+}}$microglia from 4-month-old male C57Bl6/J wild-type mice $(N=10)$ isolated using two different strategies: magnetic-activated cell sorting (MACS $N=5$, $>90 \%$ enrichment [25]) and fluorescence activated cell sorting (FACS, $N=5$, average 30,000 total cells per sample). MACS-enriched and FACS-isolated microglia cell lysates in $8 \mathrm{M}$ urea were digested with LysC and Trypsin, labeled with isobaric multiplex TMTs, and analyzed by synchronous precursor selection (SPS)-based MS3 (SPSMS3) (Fig. 1b). Due to the isobaric nature of the tags, all shared peptides from the 10 samples exhibit the same biochemical properties (i.e., exact mass, ionization efficiency, and retention time) in the MS1 or precursor scan. Only following MS/MS (or MS3-SPS) does each tag fragment and release unique reporter ions, which are then used for peptide quantitation. Thus, one major advantage of multiplex TMT based quantification is that the peptides are pooled across all samples, which increases the precursor peptide signal in the mass spectrometer for proteins common to all samples by up to 10-fold (e.g. TMT 10-plex) when compared to running each sample individually by LFQ. This is especially important when isolating small numbers of cells $(\sim 30,000)$ as in this study. In total, we identified 6404 peptides mapping to 1876 protein groups. Of these, 1791 proteins were quantified in at least 3 of the 5 replicates in each group, and 4 demonstrated expression patterns unique to only the MACS-enriched group, of which we excluded 2 due to low protein FDR. Despite the potential relative advantages or disadvantages of FACS and MACS-based microglial purification strategies, we hypothesized that core microglial proteins must be measured with high abundance by both approaches. We first focused on consensus between both the proteomes and identified 10 proteins (Hmga1, Msn, Pfn1, Myh9, Coro1a, S100a9, Cap1, Cotl1, Ctsd) that satisfied the following criteria: (1) highly abundant (>90th percentile) in MACS and FACS microglial proteomes in our data, and (2) most highly expressed at the protein level in microglia as compared to neurons and other glial cells based on an existing cell type-specific proteomic reference database [22].

Although FACS and MACS based purification of microglia yield high microglial purity ( $>90 \%$ for both), significant differences in the types of proteins identified may exist. Comparing FACS and MACS microglial proteomes, a total of 953 proteins were differentially enriched ( $p<0.05$, unpaired $\mathrm{t}$-test), and of these, 815 
met significance thresholds at the FDR $<5 \%$ level. Of the 953 differentially enriched proteins, 685 were increased and 268 proteins were decreased in abundance in the FACS microglia proteome. When we compared the relative abundance of the 685 significantly increased proteins, 36 proteins had a 2 -fold or greater increase in abundance (Fig. 2a, solid orange dots delineated by dotted line) in the FACS proteome than in the MACS proteome (top 5: Apex1, Fam3c, Lcn2, Lbr, S100a9). Of the 268 significantly decreased proteins, 65 proteins had a 2-fold or greater decrease in abundance in the FACS proteome (Fig. 2a, solid blue dots delineated by dotted line), i.e., an increased abundance in MACS proteome (top 5: Gap43, Gpm6b, Sh3gl2, Psip1, Hmgn3). Gene ontology (GO) analysis of proteins significantly decreased $(n=268)$ in the FACS microglia proteome

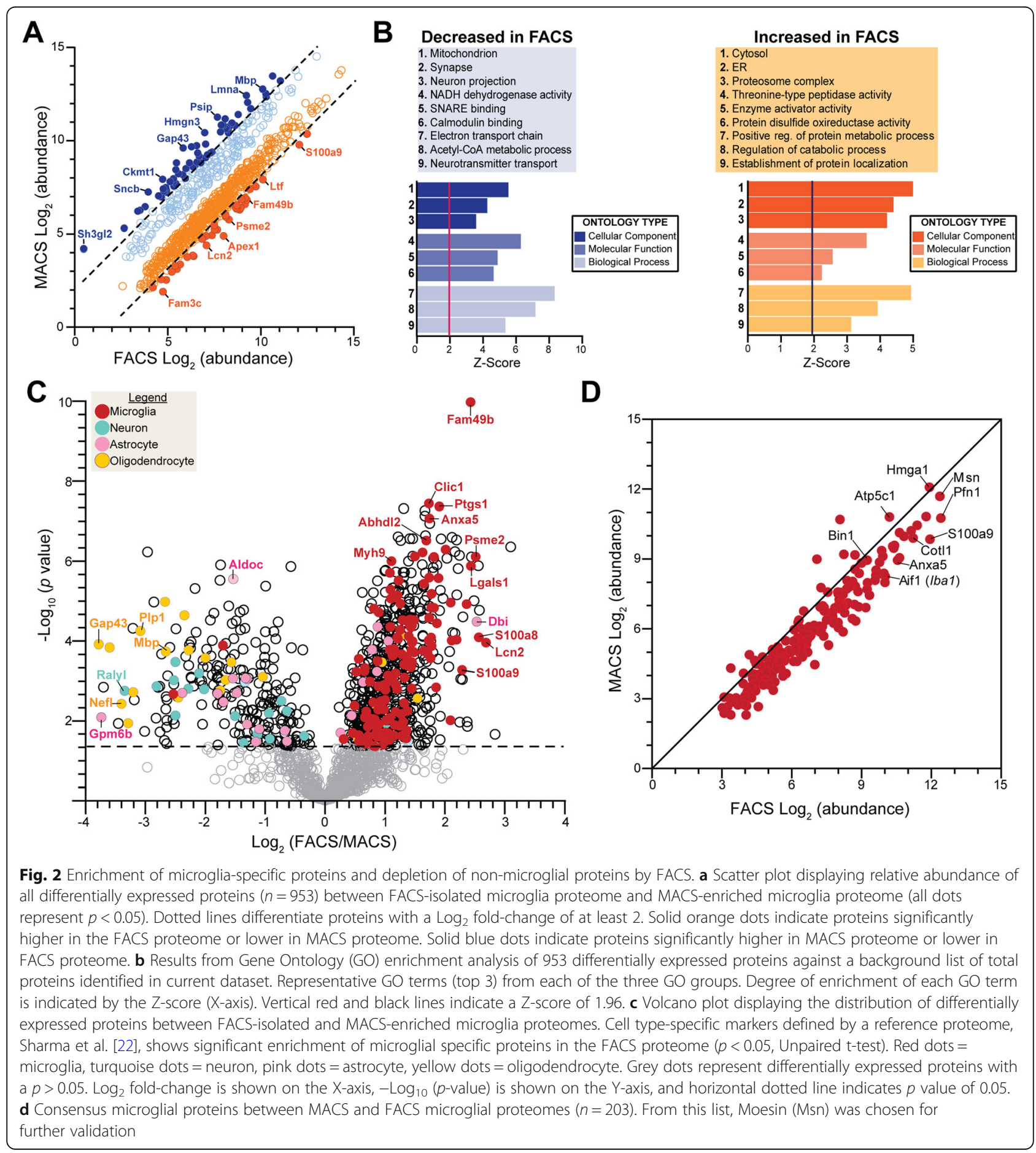


indicated an over-representation of "mitochondrial" and "synaptic" proteins, and proteins involved in "electron transport chain", "neurotransmitter transport", and "synaptic transmission" processes (Fig. 2b). Conversely, "cytosolic", "endoplasmic reticulum (ER)", and "ribosome" cellular components, and proteins involved in processes such as "regulation of protein metabolism" and "immune system" were overrepresented by the proteins significantly increased $(n=685)$ in the FACS microglia proteome (Fig. 2b). The cytosolic and ER bias of the FACS proteome, argues against a nuclear proteomic bias that could be expected using the FACS approach.

These results show that despite using identical mass spectrometry approaches and high cellular purity obtained by MACS and FACS-based microglial enrichment approaches, the proteomes from each strategy are indeed very different. Namely, the proteome of MACS-enriched microglia appears to be heavily biased towards synaptic proteins, suggesting a significant neuronal component in these samples, while the FACS-isolated proteome is enriched for immune function, indicating higher enrichment of microglial proteins. Despite these methodological differences, we identify a core set of highlyabundant microglial proteins (Fig. $2 \mathrm{~d}, n=203$ ).

\section{FACS-isolated microglial proteomes result in higher enrichment of microglial proteins and depletion of non- microglial protein contaminants}

As compared to MACS, FACS has the added advantage of confidently gating out non-cellular but protein-rich elements present in cell preparations from the brain. Based on this and our observed proteomic differences between FACS and MACS-based approaches, we hypothesized that the FACS-based strategy results in greater enrichment of microglial proteins and depletion of non-microglial proteins that may contaminate mononuclear cell preparations from the brain. Therefore, we performed cell-type enrichment analysis of differentially expressed proteins using protein marker lists derived from existing reference proteomes of four purified mouse brain cell-types - microglia, neurons, astrocytes, and oligodendrocytes [22] (Fig. 2c). Although endothelial cells represent a key brain cell population, at the time of this work, there was no existing reference for purified endothelial cell proteome. Among the 953 proteins differentially expressed comparing FACS vs MACS proteomes, we observed a marked enrichment of proteins highly expressed by microglia in the FACS proteome. The microglial proteins with high FACS-proteome abundance included Fam49b, Ptgs1, Clic1, Anxa5, and Psme2 (Fig. 2c, red solid dots). In general, microglia-specific proteins were quantified at higher abundances in FACS compared to MACS proteomes (Fig. 2d, Supplemental
Figure 2A). Conversely, neuronal, astrocytic and oligodendroglial proteins were relatively highly abundant in the MACS proteome (Supplemental Figure 2B-D).

In addition to utilizing a reference brain cell typespecific proteome [22], we further analyzed our data for cell-type enrichment using a reference transcriptomic (RNAseq) dataset of five purified mouse brain cell types - microglia, neurons, astrocytes, oligodendrocytes, and endothelial cells [11] (Supplemental Figure 2E). Similar to our observations using reference proteomic data, we observed higher representation of microgliaspecific genes among proteins enriched in the FACS proteome while neuronal, astrocytic and oligodendroglial genes were more highly represented in the MACS proteome (Supplemental Figure 2E). Interestingly, genes highly abundant in endothelial cells were also more highly abundant in the FACS proteome (Supplemental Figure $2 \mathrm{E}$, green dots). This intriguing enrichment of endothelial markers in the FACS-based microglial proteome could indicate molecular overlap between microglia and endothelial cells or could highlight limitations of prior endothelial transcriptomes. However, this can likely be further resolved by future purified endothelial proteomic studies.

Since both reference proteomic and transcriptomic cell type-specific datasets used for our analyses were independent, we assessed the degree of overlap between cell specific markers identified by these references (Supplemental Figure 2F). Interestingly, the two reference celltype marker lists we used identified minimally overlapping and varying numbers of proteins for each cell-type. For example, of the 140 microglia-specific proteins identified at the proteomic level, only 87 were also identified as enriched in microglia at the transcriptomic level (Supplemental Figure 2F). Despite these differences in reference datasets, the consensus between both our cell enrichment analyses highlights the validity of our findings regarding the advantages of the FACS approach over MACS enrichment for microglial proteomics. A detailed summary of differential representation of brain cell type-specific genes and proteins is provided in Table 1 .

To further confirm the validity of the apparent cellular biases of MACS vs. FACS proteomes in our dataset, we compared our results to our recently published MACSbased mouse microglial proteome from wild-type mice and 5xFAD mouse models in which 4133 proteins were identified by TMT-MS in $\mathrm{CD}_{11 \mathrm{~b}^{+}}$MACS-enriched microglia [25]. In this secondary analysis of an independent MACS-enriched microglial proteome, we crossreferenced the data with the reference cell-type proteomes [22] and found that $78.5 \%$ of all proteins did not map to a given cell-type, while only $4.5 \%$ were microglial and $17 \%$ of the proteins were enriched in other celltypes (Supplemental Figure 3A). While microglia- 
Table 1 Summary of cell-type enrichment in differentially expressed proteins between MACS and FACS isolated microglia proteomes

\begin{tabular}{|c|c|c|c|c|}
\hline Reference & Cell type & Increased in FACS n (\%) & Decreased in FACS n (\%) & Total $n$ \\
\hline \multirow{5}{*}{$\begin{array}{l}\text { Reference cell-type proteome } \\
\text { Sharma et al. } 2015 \text { [22] }\end{array}$} & No cell-type & $520(70.6)$ & $217(29.4)$ & 737 \\
\hline & Microglia & $139(99.3)$ & $1(0.7)$ & 140 \\
\hline & Neuron & $2(10)$ & $18(90)$ & 20 \\
\hline & Astrocyte & $19(57.6)$ & $14(42.4)$ & 33 \\
\hline & Oligodendrocyte & $5(21.7)$ & $18(78.3)$ & 23 \\
\hline \multirow{6}{*}{$\begin{array}{l}\text { Reference cell-type transcriptome } \\
\text { Zhang et al. } 2014 \text { [11] }\end{array}$} & No cell-type & $515(71.3)$ & $207(28.7)$ & 722 \\
\hline & Microglia & $119(100)$ & 0 & 119 \\
\hline & Neuron & $4(8)$ & $46(92.0)$ & 50 \\
\hline & Astrocyte & $7(43.8)$ & $9(56.2)$ & 16 \\
\hline & Oligodendrocyte & $11(73.3)$ & $4(26.7)$ & 15 \\
\hline & Endothelial cell & $29(93.5)$ & $2(6.5)$ & 31 \\
\hline
\end{tabular}

specific proteins such as Msn and Cotl1 were highly abundant in the MACS-enriched microglial proteome, neuronal proteins (Camk2a, Gap43), astrocyte proteins (Gfap, Aldoa) and oligodendrocyte proteins (Mbp) were also identified as equally highly abundant proteins (Supplemental Figure 3B, >90th percentile of relative abundance). Non-microglial proteins were also found in the FACS-isolated microglial proteome at relatively high abundance (Supplemental Table 1). For example, Mbp (90th percentile), Gfap (60th percentile), Camk2a ( $>75$ th percentile) were still relatively abundant in the FACS microglial proteome, although many fold lower compared to the MACS proteome where these three proteins were found at $>90$ th percentile of abundance.

Collectively, these cell-type enrichment analyses of our proteomic data show that MACS-based proteomes are still contaminated by non-microglial proteins despite high microglial cellular purity likely due to remnant acellular debris; while FACS-based microglial isolation results in a purer microglial proteome that is less contaminated by neuronal, astrocytic and oligodendroglial proteins. The presence of non-microglial proteins at relatively high abundance in the FACS-isolated microglial proteome may be more indicative of phagocytic uptake on non-microglial cellular elements by microglia [45]. Another interesting finding is that a majority of proteins expressed in microglia are not specific to microglia but are shared across various cell types.

\section{Cotl1 and Msn are highly expressed by microglia in adult mouse brain}

Based on our consensus findings between different microglia enrichment strategies, we have defined a core set of highly-abundant microglial proteins (Fig. 2 d, $n=203$ ) in adult mice which can be used as indicators of microglial abundance in future proteomic studies. Among proteins with high concordance in microglia, several proteins including Msn, Cotl1 and Bin1 are identified to be highly abundant in both FACS and MACS proteomes (Fig. 2d). We chose Cotl1 and Msn for additional neuropathological studies to characterize expression in the mouse brain. Coactosin like F-actin binding protein 1 (Cotl1) is an actin-binding protein expressed by immune cells including macrophages and has been recently reported to regulate actin dynamics at the immune synapse [46, 47]. Cotl1 along with Msn also bind polyunsaturated fatty acid chemokines [48]. Furthermore, Cotl1 is highly and specifically expressed by microglia at the transcript and protein levels $[11,22]$ we have previously identified as a novel microgliaspecific marker from a quantitative TMT-MS proteomic analysis of MACS-enriched $\mathrm{CD}_{11 \mathrm{~b}^{+}}$microglia of adult mice in normal, acute neuroinflammatory, and chronic neurodegenerative states [25]. In the current study, we were able to replicate our previous finding of Cotl1 as a microglial marker with a significantly higher abundance (1.4-fold higher, $p=0.001$ ) in the FACS proteome than MACS proteome. We immunostained brains from 6 to 7 month old Cx3cr1 ${ }^{\text {CreER-YFP }}$ mice on wild-type (WT) or $5 x F A D$ backgrounds with antibodies against YFP/GFP, to detect microglia, and Cotl1. Cx3cr1 ${ }^{\text {CreER-YFP }}$ mice express YFP/GFP immunofluorescence in $\mathrm{Cx}_{3} \mathrm{Cr}^{+}$microglia in the brain $[33,34]$. In the $C \times 3 \mathrm{Cr}^{\mathrm{CreER}-\mathrm{YFP}}-\mathrm{WT}$ and Cx3cr1 ${ }^{\text {CreER-YFP }}-5 x F A D$ cortices, Cotl1 immunofluorescence was specifically detected in GFP immunofluorescent microglia (Supplemental Figure 4A, arrow). The $\mathrm{GFP}^{+}$ microglia in the Cx3cr1 ${ }^{\text {CreER-YFP }}$-WT cortex also appeared morphological ramified, but those in $\mathrm{Cx} 3 \mathrm{cr} 1^{\text {CreER-YFP }}$ $5 \mathrm{xFAD}$ cortex adopted an activated morphology with shorter swollen processes and larger cell bodies (Supplemental Figure 4A). We have previously described a similar morphological phenotype in a WT and 5xFAD mouse brains as well as demonstrated a quantitative increase of Cotl1 expression in 5xFAD brain compared to WT brain [25]. 
Moesin (Msn) is a member of the ezrin-radixinmoesin (ERM) family of proteins that link the Cterminal domain of cortical actin filaments to the plasma membrane [49]. Outside the brain, Msn in found in other tissues and cell types including macrophages, lymphocytes, epithelial cells, and endothelial cells [50-52]. In the brain, Msn is most abundantly expressed by microglia compared to neurons, oligodendrocytes or astrocytes although a brain endothelial cell proteome is currently lacking [22]. At the transcript level, Msn is highly expressed by endothelial cells and microglia [10, $11,22]$. We immunostained the brains from Cx3cr1 ${ }^{\text {CreER-YFP }}$ mice on WT or $5 x F A D$ backgrounds for YFP/GFP (to detect microglia) and Msn. In CX3cr1 ${ }^{\text {CreER-YFP }}$-WT mouse cortex, we observed GFP immunofluorescence within the cell body and processes of ramified microglia (Fig. 3a, arrow) and detected diffuse Msn immunofluorescence in the same ramified microglia (Fig. 3a, arrow). We also observed Msn immunofluorescence in non-microglial cells which resembled endothelial cells (Fig. 3a, asterisk), consistent with previously reported Msn expression in endothelial cells [11]. In Cx3cr1 $1^{\text {CreER-YFP }}-5 x F A D$ mouse cortex, we observed GFP immunofluorescent microglia with an activated morphology and found strong Msn expression in the same microglia (Fig. 3a, arrow). We also observed GFP and Msn immunofluorescent microglia surrounding dense $A \beta$ plaques (Fig. $3 \mathrm{~b}$, arrow) in $\mathrm{Cx} 3 \mathrm{cr} 1^{\text {CreER-YFP }}$ $5 x F A D$ mouse cortex. Importantly, 5xFAD mice displayed a significant increase in Msn immunofluorescence in the cortex compared to control mice (Supplemental Figure 4B, $p<0.0001$ ). Given that Msn is abundant in endothelial cells at the transcript level and the presence of GFP negative, Msn immunofluorescent non-microglial cells (Fig. 3a, asterisk), we stained brain tissue from 9 to 10 month old WT and 5xFAD mouse brains to detect CD31 (an endothelial marker) and Msn. In both WT and 5xFAD cortices, we observed CD31 and Msn immunofluorescence in endothelial cells (Fig. 3c, arrows) as well as cells immunofluorescent for Msn only (Fig. 3c, asterisk). Additionally, we stained the same brain sections with GFAP, to detect astrocytes, and Msn. In WT and 5xFAD cortices, GFAP immunofluorescent astrocytes (Fig. 3d, asterisk) did not display Msn immunofluorescence (Fig. 3d, arrow). Overall, while Msn is found in both ramified microglia and endothelial cells in normal brain and in regions distant from $A \beta$ plaques, Msn expression is nearly exclusively found in microglia that surround $\mathrm{A} \beta$ plaques in $5 \mathrm{xFAD}$ mice.

These results validate two highly-abundant microglial specific proteins, namely Cotl1 and Msn, which can serve as markers of microglia in the mouse brain at normal and disease states and indicate possible roles for these proteins in microglial function under homeostatic and disease conditions.

\section{Msn regulates $A \beta$ phagocytosis and pro-inflammatory cytokine production by microglia}

Based on our findings of Msn expression in mouse microglia distant and proximate to $A \beta$ plaques in the $5 x F A D$ mouse brain, we performed in-vitro functional studies using primary mouse microglia to determine the effect of Msn knockdown (KD) on microglial phagocytic properties (Fig. 4a). Msn siRNA resulted in $>90 \%$ reduction in Msn mRNA expression compared to control siRNA (Fig. 4b, $p<0.001$ ). Primary neonatal mouse microglia were incubated with fluorescent-conjugated fA $\beta 42$ for $60 \mathrm{~min}$ and phagocytic uptake by $\mathrm{CD} 45^{+}$live microglia was assessed by flow cytometry [26]. Msn siRNA pre-treatment reduced $A \beta$ phagocytic uptake by $\sim 25 \% \quad(p=0.0274)$ without affecting viability as compared to siRNA control under resting conditions (Fig. 4b). In the presence of LPS stimulation, we observed minimal effect of Msn siRNA (Fig. 4b), suggesting context-dependent functional roles for Msn in microglia. In this assay, we have previously shown that inhibition of actin-dependent processes by cytochalasin D completely inhibits $A \beta$ uptake, confirming actin-dependent phagocytic uptake of $A \beta$ rather than passive binding to the cell surface [26]. Next, we measured levels of cytokines and chemokines by Luminex assays in the culture supernatants derived from primary microglia that were treated with sham siRNA or siMsn for $24 \mathrm{~h}$ and then treated with LPS (or sham) for another $24 \mathrm{~h}$ as a strong pro-inflammatory challenge (Fig. 4a). As expected, LPS increased levels of TNF, IL-10, and other cytokines (Fig. 4d-i, IL6, G-GSF, IP-10/CXCL10, and MIP-1 $\alpha / C C L 3)$. Specifically, Msn siRNA enhanced LPS-induced increase in IL-10 (Fig. 4d) and TNF (Fig. 4f, $p<0.05$ ) levels, although the former was not statistically significant. In sum, our in-vitro studies indicate that deletion of Msn from microglia impairs their ability to phagocytose $A \beta$ and increases IL-10 and TNF- $\alpha$ release after an inflammatory stimulus.

\section{Msn is a hub protein in a disease-associated proteomic co-expression module in human $A D$ brain}

In a recent large proteomic analysis of over 400 human post-mortem brains, we identified a consensus network of protein co-expression modules with robust associations to $\mathrm{AD}$ pathologies as well as cognitive traits [40]. In this network analysis, we identified a protein module (M4) that was highly correlated with higher neuropathological burden and worse cognitive outcomes; this module was highly enriched in microglial and astrocytic proteins [40]. Msn is a hub protein, defined as a protein most central to module function with a high module membership value (kME), of this module (Fig. $5 \mathrm{a}, \mathrm{kMe}=$ 0.807 ) as well as several other microglial proteins identified in the current mouse microglial proteomic analysis 


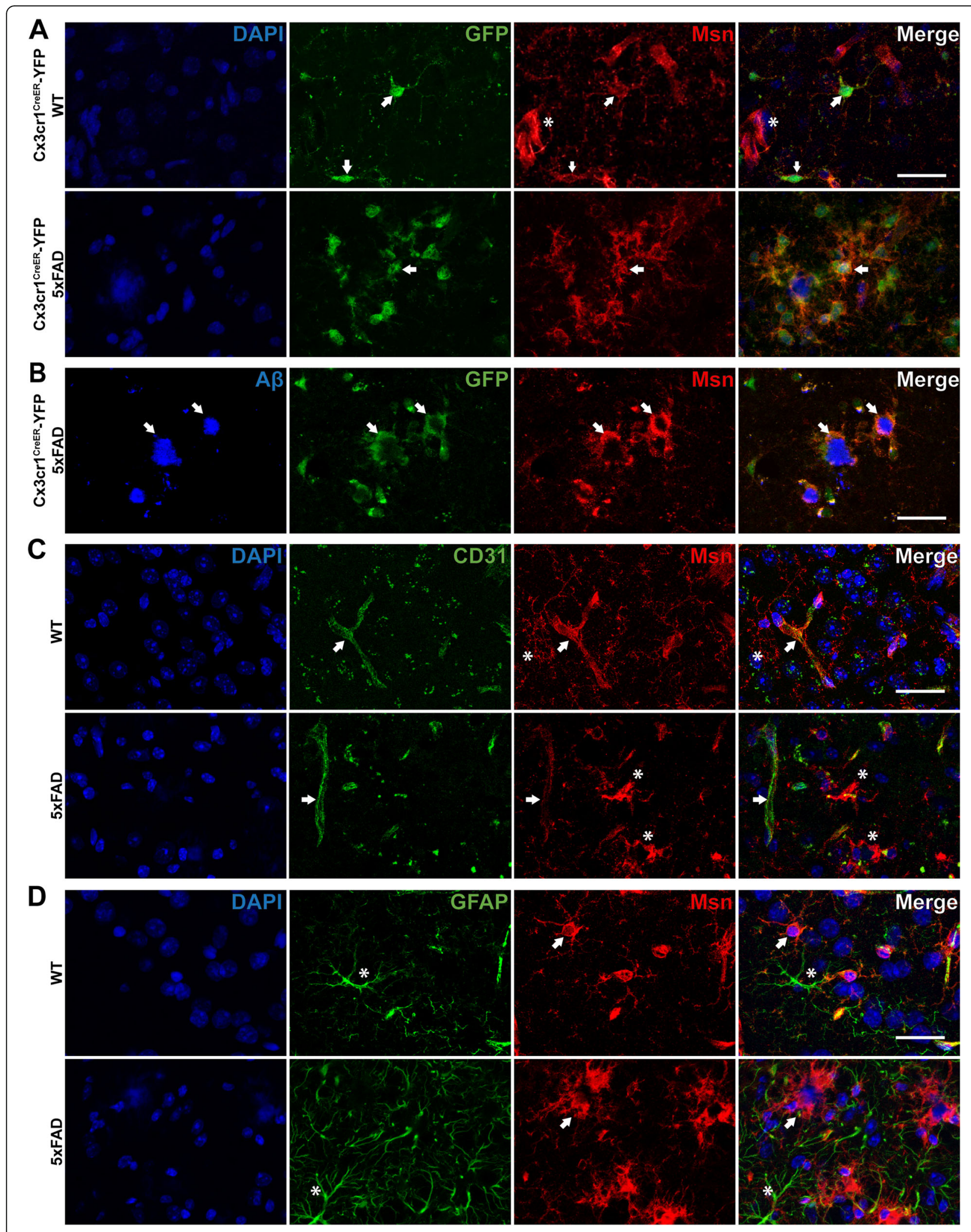

Fig. 3 (See legend on next page.) 
(See figure on previous page.)

Fig. 3 Moesin is expressed by microglia and endothelial cells in mouse brain. a Representative immunofluorescence images of Cx3Cr1 ${ }^{\text {CreER-YFP }-W T}$ $(N=4)$ and $C \times 3 \mathrm{Cr}_{1}{ }^{\text {CreER-YFP }}-5 \times$ XAD $(N=6)$ mouse cortex stained for microglia (GFP) and Msn. Arrow indicates microglia immunopositive for GFP

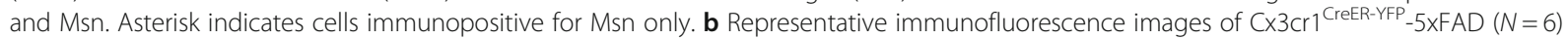
mouse cortex stained for amyloid-beta (A $\beta$ ), microglia (GFP), and Msn. Arrow indicates A $\beta$ plaque as well as microglia immunopositive for GFP and Msn. c Representative immunofluorescence images of WT $(N=3)$ and 5xFAD $(N=4)$ mouse cortex stained for endothelial cells (CD31) and Msn. Arrow indicates endothelial cells immunopositive for CD31 and Msn. Asterisk indicates cells immunopostive for Msn only. d Representative immunofluorescence images of WT $(N=3)$ and $5 x$ FAD $(N=4)$ mouse cortex stained for astrocytes (GFAP) and Msn. Arrow indicates cells immunopositive for Msn only. Asterisk indicates astroctyes immunopostive for GFAP only. Scale bar $=30 \mu \mathrm{m}$

(Fig. 5a, red circles: ANXA5, CRYL1, TPP1, COTL1, CTSB, and CAP1). Msn protein abundance was significantly elevated in AD DLPFC compared to control or AsymAD DLPFC (Fig. 5b, $p<0.001$ ). Furthermore, increased Msn levels were also present in AD brains even at the transcript level (Fig. 5c, $p<0.001$ ) [41]. We observed that Msn protein abundance displayed a strong negative correlation with cognitive function (Fig. $5 \mathrm{~d}$, MMSE, $r=-0.51, p=1.9 \mathrm{e}-12$ ). Cognitive function was determined by the mini-mental status examination (MMSE) score where higher scores $(>24)$ represent better cognitive function and lower scores $(<24)$ represent cognitive dysfunction (or dementia) [53]. Furthermore, Msn protein abundance demonstrated a strong positive correlation with $\mathrm{A} \beta$ plaque burden (Fig. 5d, CERAD, $r=$ $0.32, p=2 \mathrm{e}-11$ ) and neurofibrillary tau tangle pathology (Fig. 5d, BRAAK, $r=0.33, p=4.2 \mathrm{e}-12$ ) [54, 55]. We further characterized Msn expression via immunohistochemistry and observed a morphological difference between control and $\mathrm{AD}$ brain sections (Fig. 5e). Msn immunoreactive cells in the control brain showed a typical, ramified microglial morphology (Fig. 5e, Control inset), while Msn immunoreactive cells resembled activated microglia in the $\mathrm{AD}$ brain (Fig. 5e, $\mathrm{AD}$ inset).

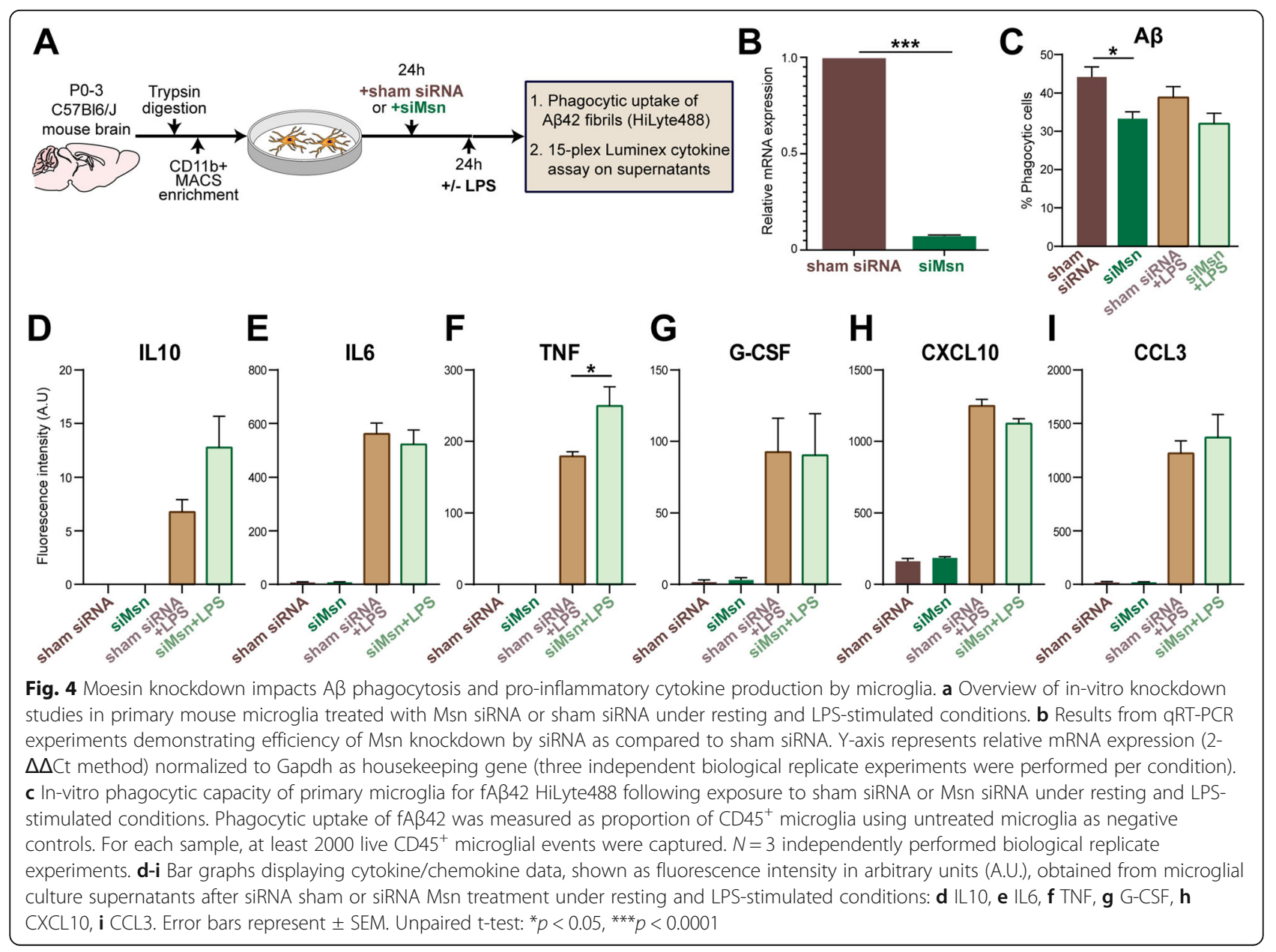




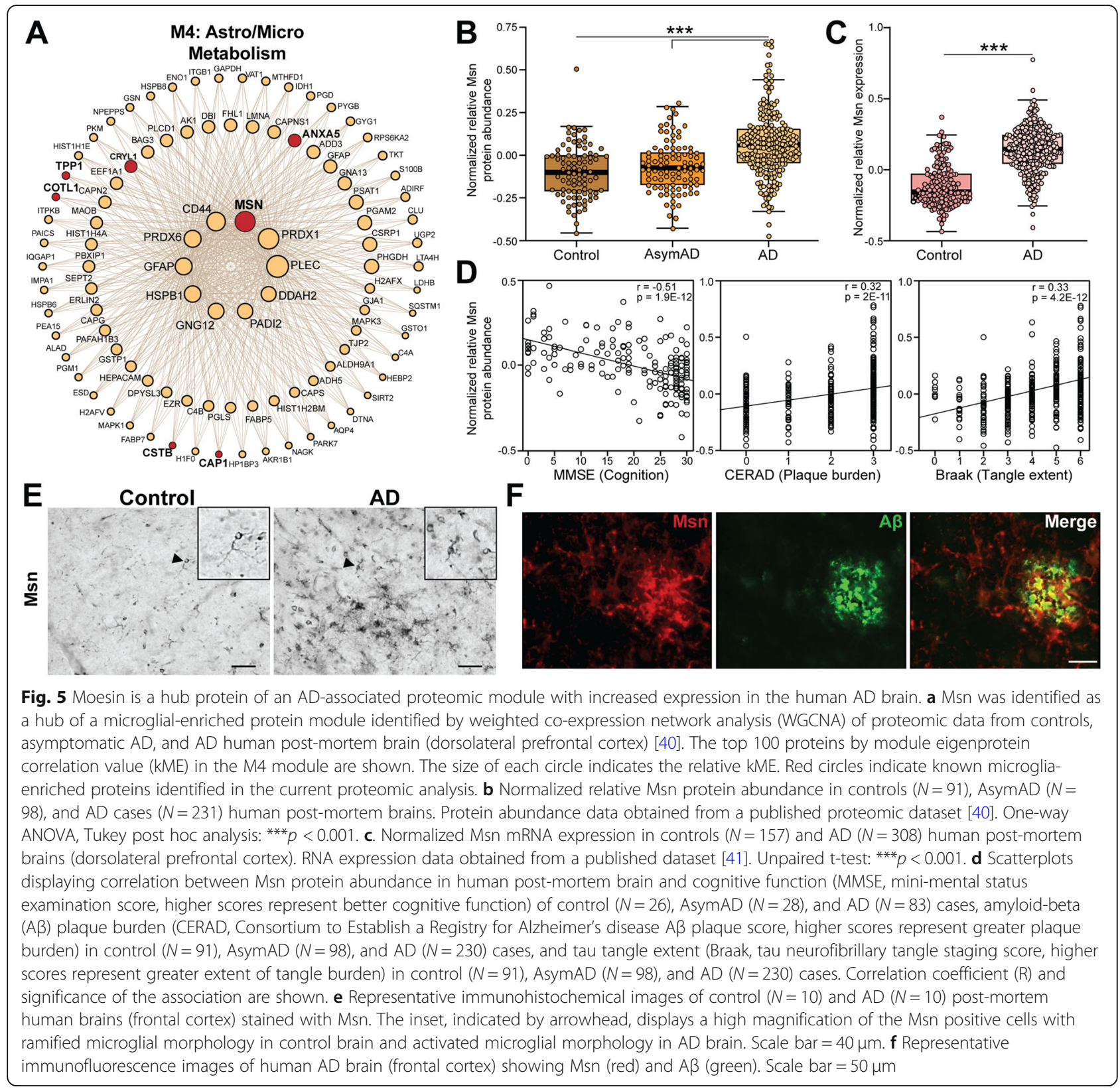

Lastly, we observed Msn immunofluorescent cells to be surrounding $A \beta$ plaques in $A D$ brain (Fig. $5 \mathrm{f}$ ), similar to what we observed in the 5xFAD mouse brain (Fig. 3b).

Using independent proteomic datasets, we examined Msn protein abundance in published human postmortem brain cohorts which included a variety of neurodegenerative disease cases $[40,42]$. Similar to our primary findings (Fig. 5b), Msn abundance was significantly increased in AD DLPFC compared to controls in an independent cohort (Supplemental Figure 5A, $p<0.001$ ). Interestingly, we observed a significant elevation in Msn abundance in FTLD-TDP DLPFC compared to controls (Supplemental Figure 5A, $p<0.001$ ); however, this elevation was not seen in ALS and PD/PDD brains (Supplemental Figure 5A). Next, we wanted to determine whether Msn levels were increased in a different brain region other than DLPFC; thus we analyzed a proteomic analysis of the precuneus in control, AsymAD, and $\mathrm{AD}$ cases [40]. We chose this region because the precuneus is affected early in the course of $\mathrm{AD}$, as shown by clinical, imaging, and pathological studies [56]. We found Msn to be significantly elevated in AD precuneus compared to control (Supplemental Figure 5B, $p<0.01$ ) and asymptomatic (Supplemental Figure 5B, $p<0.05$ ) cases. There was an elevation of Msn levels in AsymAD precuneus compared to controls, although it was not 
statistically significant. Lastly, since Msn expression is observed in microglia proximate to $A \beta$ plaques in human $\mathrm{AD}$ and $5 \mathrm{xFAD}$ mouse brains, we sought to determine Msn protein abundance in a published proteomic analysis of laser-micro-dissected $\mathrm{A} \beta$ plaques from the hippocampus and adjacent entorhinal cortex of rapidly progressive $\mathrm{AD}(\mathrm{rpAD})$ and sporadic $\mathrm{AD}(\mathrm{spAD})$ cases [17]. We found Msn abundance to be significantly lower in rpAD cases compared to spAD cases (Supplemental Figure $5 \mathrm{C}, p<0.001$ ) showing that despite the observed increased Msn expression in AD, it appears to have a negative association with rapidly progressive $\mathrm{AD}$.

In addition to exploring human proteomic datasets, we examined published TMT-MS and RNAseq data for Msn protein and transcript levels, respectively, in mouse models of AD pathology [43, 44]. Quantitative proteomics using TMT-MS was conducted on brains from WT, 5xFAD (A $\beta$ pathology), JNPL3 (tau pathology), and a cross of $5 x F A D$ and JNPL3 (A $\beta$ \& tau pathologies) mice at 4 months, 7 months, and 10 months of age. There was an age dependent increase in Msn protein abundance in the 5xFAD and 5xFAD/JNPL3 brains compared to age-matched WT brains (Supplemental Figure 6A, $p<0.001$ ), while Msn protein abundance was unchanged in JNPL3 mice. We observed a similar age dependent increase in Msn transcript levels in 5xFAD brain compared to WT brains, with a significant increase at 12 months of age (Supplemental Figure 6B, $p<0.05$ ). Overall, analysis of these independent human and mouse proteomic and transcriptomic studies increases the validity of our finding of increased Msn in human AD and highlights a potential novel role for Msn in FTLD-TDP pathology.

\section{Discussion}

Studies of human brain gene and protein expression have consistently identified microglial genes/proteins within immune pathways as determinants of disease progression and cognitive decline [3, 30, 31]. Molecular characterization of microglia has been traditionally biased towards transcriptomic studies $[41,57,58]$ rather than proteomic studies due to the generally low protein yield from isolated cells, challenges related to microglial isolation from the brain, and technical requirements for mass spectrometry analyses. Multiple studies have shown the discordance between transcript-level and protein-level expression data attributed to posttranscriptional processes such as post-transcriptional mRNA regulation, post-translational protein modifications, protein recycling and degradation [19-21]. Therefore, comprehensive profiling of proteins, rather than transcripts, of microglial cells is necessary for a deeper understanding of microglia-mediated disease mechanisms in neurodegenerative diseases such as AD. Prior proteomic studies have used magnetic-activated cell sorting (MACS) enrichment strategies to isolate microglia from fresh mouse or human brain [22, 25, 59]. MACS-enrichment aims to facilitate a rapid, highthroughput, immuno-magnetic separation of a pure population of a desired cell-type, i.e. microglia, from a single cell-suspension; however, it is limited by contamination by non-microglial proteins despite high cellular purity.

In this first FACS-based microglial proteomics study in adult wild-type mice, we have established the feasibility of this approach for microglial proteomics and have demonstrated that FACS-based microglial enrichment is the approach of choice for microglial proteomics studies. As compared to the MACS strategy, FACS effectively gates out protein-rich debris in the preparation which are likely cellular remnants of other cells such as neurons, astrocytes and oligodendrocytes that are enriched along with microglia due to the cellular complexity of the brain. Since non-cellular elements are likely to be non-nucleated, FACS or MACS approaches can be equivalent approaches for transcriptomic studies due to high cellular purity. However, proteomics studies are easily skewed by proteins in non-cellular elements in the preparation. As a result of this advantage of FACS, the proteomes obtained by each isolation strategy are indeed very different: the MACS-enriched microglia proteome over-represents synaptic proteins, suggesting a significant neuronal component in these samples, while the FACS-isolated microglia proteome is enriched for immune function proteins, indicating higher enrichment of microglial proteins. In order to assess whether the FACS proteome was indeed enriched for microglial proteins, we performed cell-type enrichment analysis of the differentially expressed proteins between the MACS and FACS proteomes and demonstrated that the FACSisolation approach, when coupled with TMT-MS, is a superior method yielding a proteome that is highly enriched for canonical microglia-specific proteins while non-microglial proteins, particularly those derived from neurons and oligodendrocytes, are significantly depleted.

A strength of our study is the use of TMT-MS for quantitative proteomic characterization. The multiplex paradigm enables the accurate quantitation of thousands of proteins across many samples simultaneously for large-scale quantitative proteomic applications $[60,61]$. Also, a major advantage of TMT-MS is the ability to multiplex all of the peptide sets prepared from multiple samples to be combined into a single LC-MS/MS analysis, resulting in improved breadth of coverage by minimizing missing values that are common in 'shotgun' label-free based quantification [28, 62]. The combination of TMT-MS and SPS-MS3 methods significantly improved the acquisition, and quantitation of MACS- 
enriched and FACS-isolated microglial proteomes. Critically, collapsing the 10-TMT channels (5 samples/isolation strategy) resulted in higher signals and identified proteins, overcoming the challenges of low protein yield typically expected from isolation of rare cell-types such as microglia. Many studies pool isolated microglia from 3 to 12 mouse brains for a MS run to increase protein yield and improve detection/quantitation of proteins in a MS run [11, 22, 25]; however, each sample in our study consisted of microglial cells isolated from one whole mouse brain without pooling for either isolation strategy. This is significant because 1) we demonstrate that at least 18,000 FACS-isolated microglia from one mouse brain is sufficient for a thorough proteomic analysis, 2) sampling bias is minimized when samples are not pooled, and 3) this method is especially cost-effective for complex, long-term mouse studies. The limitations of our approach are also made apparent by the aforementioned points. We identified approximately 1800 proteins compared to the 4133 proteins identified in our previous proteomic analysis of acutely isolated microglia [25]. This low number of identified proteins could have been ameliorated by pooling brain samples, as this significantly increases net protein yield. Additionally, enzymatic digestion rather than mechanical dissociation of brain tissue prior to isolation could increase microglial cell yield from one whole brain, and thus, protein yield. Notably, mechanical subcellular dissociation coupled to density-based centrifugation for purification of intact nuclei from brain for various downstream applications including FACS-MS has been demonstrated to achieve high purity separation of neuronal from non-neuronal nuclei [63] and this approach could incorporate detection of nuclear microglial-specific marker proteins from the current study to purify microglial nuclei. Lastly, the increased number of protein isoforms found by MACS could be attributed to non-cellular contamination in the MACS-only samples.

Our study also supports the use of our validated pipeline of FACS-based isolation coupled with TMT-MS for characterization of non-microglial brain cell types. One advantage of the FACS approach is that it provides the opportunity to isolate other cell-types from the brain, which is not feasible using MACS approaches without compromising cell integrity and viability. In our current study, we did not perform enzymatic digestion of the brain to maximize microglial enrichment prior to FACS or MACS. Enzymatic digestion can significantly increase the yield of endothelial cells as well as other glial cells (astrocytes and oligodendrocytes), allowing simultaneous isolation of multiple cell types by FACS. Recently, a cell isolation methodology termed concurrent brain cell type acquisition (CoBrA) has been used to isolate microglia, endothelial cells, astrocytes, and oligodendrocytes from mouse brain for RNAseq studies [64]. Unlike transcriptomic approaches, proteomic applications require specific strategies to reduce protein contamination from serum, albumin, and other proteins such as keratin, which we are currently optimizing for an analogous simultaneous cell-type isolation pipeline for proteomic studies. Based on our demonstration of a high-quality proteome from FACS-isolated microglia, which traditionally have very low protein yield per cell, our results support the feasibility of using FACS to isolate distinct cell types with high purity while minimizing non-specific contamination, for simultaneous proteomic characterization of multiple cells types. This is particularly important for resolving why we observed a significant enrichment of endothelial genes/proteins in our FACS-isolated microglial proteome, which could mainly be a reflection of our cell-type enrichment analysis method for endothelial cells. The proteomic reference markers suffer from the absence of an endothelial proteomic profile, by which markers that are common to both microglia and endothelia could be excluded by the thresholding rubric performed to define the cell-type enriched marker lists. Current brain endothelial cell biology is solely based on expression at the transcript level because no purified endothelial proteomes exist, thus, our cell-type enrichment analysis was conducted using a mouse brain cell-type transcriptomic reference [11]. Given the discordance between transcript-level and protein-level expression, it is difficult to conclude that there is indeed endothelial cell-type protein enrichment by FACS targeting microglial enrichment. This can only be resolved by proteomic characterization of concurrently isolated microglial cells and endothelial cells from the same mouse brain and comparison of these data with existing endothelial transcriptomic data.

We assessed our MACS- and FACS- microglial proteomes for cell-type enrichment with two different mouse brain cell-type reference datasets: cell-type resolved proteome by Sharma et al. [22] and cell-type resolved transcriptome by Zhang et al. [11]. Although, both reference datasets identified the FACS-proteome to be enriched with microglial proteins/gene symbols and depleted of non-microglial proteins/gene symbols, there were still a significant number of gene products that were not assigned to a specific cell-type. This could be attributed to several factors. First, it could be that these proteins/genes are ubiquitous in the brain regardless of the cell-type. Second, there are inherent differences in the isolation methodologies between our study and the reference studies. For example, the reference cell-type proteome [22] isolated cells using MACS-enrichment, while the reference cell-type transcriptome [11] utilized FACS isolation from transgenic mice and immunopanning to isolate cells. Lastly, the expression or regulation 
of these proteins/gene symbols in their respective celltype might vary with age. We characterized the microglial proteome of adult mice, while both reference datasets characterized microglia and other brain cell-types from vastly younger mice ranging in age from P1 to P8* [11, 22]. In summary, the combination of all these factors may have limited our ability to fully resolve the cell-type origin of the proteins identified within our dataset and highlights the need for comprehensive characterization of the proteome or transcriptome of mouse brain cell-types using consistent mouse models at similar ages, isolation protocols, and technical and analytical measures that will allow for cross-study comparisons.

Despite the advantages of the FACS approach, highlyabundant microglia proteins should still be identified using either strategy. Therefore, using a consensus between both approaches, we identified several proteins with high abundance in both datasets, and the top 10 proteins of this list were expressed in both datasets at or above the 90th percentile of abundance. Of these highly-abundant and microglia-specific proteins, we considered Msn and Cotl1 for validation studies. Cotl1 protein expression was observed in microglia of WT and 5xFAD brains, with higher immunofluorescence and increased microglial size observed in the $5 \mathrm{xFAD}$ mice only, consistent with our prior observations [25]. The findings pertaining to Msn represent novel observations for this protein in mouse models of AD pathology and human AD. In WT mice, Msn was expressed in ramified/homeostatic microglia as well as non-microglial cells that resembled endothelial cells, consistent with prior transcriptomic studies showing that Msn is also highly abundant in endothelial cells [11]. In the 5xFAD mouse brains, Msn protein expression was localized to large clusters of reactive microglia rather than ramified microglia or endothelial cells, specifically within microglial processes, that surround and infiltrate $A \beta$ plaques, suggestive of the disease-associated microglia (DAM) phenotype that was identified previously [13]. Similar to our findings in 5xFAD brains, we also found increased Msn expression in the vicinity of $A \beta$ plaques in human $\mathrm{AD}$ brain. Consistent with ezrin-radixin-moesin (ERM) proteins, the pattern of Msn expression in microglia appeared to be more membrane localized rather than cytosolic [65]. These immunohistochemical studies show that Msn and Cotl1 proteins, like other known markers such as Tmem119 or Iba1, could serve as novel markers of microglia in the mouse brain in normal and disease states.

In a large human brain network built using over 400 frontal cortex samples, Msn was found to be a hub of a protein co-expression module that was strongly associated with neuropathology and cognitive decline, indicating a key role for Msn in AD pathogenesis. Furthermore, we found Msn protein abundance was significantly elevated in AD DLPFC and precuneus. We also found that Msn around plaques is lower in rapidly progressive $\mathrm{AD}$ brains as compared to sporadic AD brains. Our in-vitro data, combined with correlative data from human brain studies, support the idea that Msn in microglia may play important roles in $\mathrm{AD}$ pathogenesis by impacting phagocytosis and pro-inflammatory cytokine release. We identified that siRNA-mediated knockdown of Msn augmented the release of TNF and IL-10 by primary mouse microglia in response to LPS stimulation TNF or tumor necrosis factor is a pro-inflammatory molecule $[66,67]$ while $\mathrm{IL}-10$, although traditionally considered anti-inflammatory, has been found to have detrimental effects in AD models [68, 69]. While we observed an increase in inflammatory cytokine release by microglia following Msn knockdown, we also observed an increase of Msn in AD cases, and increase in TNF in AD has been previously demonstrated [68]. One very likely explanation for this apparent discrepancy between our in-vitro studies and human AD findings is that we used a pure microglial culture system without any other cell-types that might impact TNF levels or be a source of TNF itself. Indeed, in the brain, there are multiple sources of TNF, such as astrocytes, sub-populations of neurons, and even different populations of microglia [69-72]. Furthermore, these experiments do not account for the microglial heterogeneity observed in in-vivo $A D$ models [73], making it difficult to know whether loss of Msn in this context will also impact TNF release in a similar manner. Msn silencing also decreased the ability of microglia to phagocytose $A \beta$ fibrils. The role of Msn in phagocytosis is not unexpected because Msn belongs to the family of ERM proteins which are involved in actinassociated biological processes [49, 51, 52, 65]. Altogether, these findings raise the possibility that increased expression of Msn in AD may represent a compensatory and potentially protective mechanism that is most likely explained by increased microglial activation. Future studies using conditional Msn deletion in microglia or endothelial cells in mouse models of AD pathology will clarify the protective or detrimental role of Msn in AD pathogenesis. Based on our in-vitro findings of Msn deletion in microglia, additional mechanistic studies are also needed to define the molecular and cellular defects responsible for impaired $A \beta$ phagocytosis in Msn-deficient microglia.

\section{Conclusion}

In conclusion, this study establishes the feasibility and superiority of the FACS-based TMT-MS approach for quantitative proteomics of acutely-isolated microglia from adult mouse brain which can now be applied for future quantitative proteomic studies of adult mouse microglia using disease models. Using this strategy, we defined a core set of highly abundant microglia-specific proteins. Among these, we provide novel evidence for a significant and potentially protective role for Msn in human AD. 


\section{Supplementary information}

Supplementary information accompanies this paper at https://doi.org/10. 1186/s13024-020-00377-5.

Additional file 1: Table S1. Quantitative protein expression data from MACS-enriched and FACS-isolated mouse microglia. Log 2 transformed protein abundance data and differential expression analysis.

Additional file 2: Table S2. GO Elite analysis of 953 significantly differentially expressed proteins between MACS-enriched and FACSisolated mouse microglia proteomes.

Additional file 3: Figure S1. Viability of mechanically dissociated mouse brain mononuclear cells. Representative flow cytometry data displaying isolation of $>95 \%$ live CD $11 b^{+}$microglia from mechanically dissociated fresh, whole mouse brian $(N=4)$ following percoll density centrifugation.

Additional file 4: Figure S2. Enrichment of microglial and endothelial specific proteins by FACS. A-D Histograms displaying top 10 differentially expressed A microglia, B neuron, C astrocyte, and D oligodendrocyte cell-type proteins (defined by Sharma et al. [22]) in FACS-isolated microglia proteome and MACS-enriched microglia proteome. The Y-axis shows list of proteins, $X$-axis shows Log $_{2}$-transformed normalized abundance (abundance/row geomean), and error bars represent \pm SEM. E Volcano plot displaying the distribution of differentially expressed proteins between FACS-isolated and MACS-enriched microglia proteomes. Cell-type enrichment defined by a reference cell-type transcriptome, Zhang et al [11], shows significant enrichment of microglial and endothelial specific proteins in the FACS proteome ( $p<0.05$, Unpaired t-test). Red dots $=$ microglia, turquoise dots $=$ neuron, pink dots $=$ astrocyte, yellow dots $=$ oligodendrocyte, green dots = endothelial cell. Grey dots represent differentially expressed proteins with a $p>0.05$. $\log _{2}$ fold-change is shown on the $X$-axis, $-\log _{10}(p$-value) is shown on the $Y$-axis, and horizontal dotted line indicates $p=0.05$. F Venn diagrams comparing number of cell-type specific proteins defined by our two cell-type enrichment analyses: reference cell-type proteome: Sharma et al. [22] and reference cell-type transcriptome: Zhang et al. [11].

Additional file 5: Figure S3. Contamination of non-microglial proteins in MACS-enriched microglia proteome. A Pie chart displaying distribution of cell-type enrichment in previously published microglial proteome, Rangaraju et al. [25], where $4.5 \%$ of the 4133 quantified proteins are microglial-specific while nearly $17 \%$ of the proteins are from other brain cell-types. B Highly-abundant proteins in a MACS microglial proteomic study [25] included non-microglial proteins such as Mbp, Aldoa, Gfap, and Camk2a. Micro = microglia, Neu = neuron, Astro = astrocyte, Oligo = oligodendrocyte.

Additional file 6: Figure S4. Validation of Cotl1 in microglia. A Representative immunofluorescence images of $\mathrm{Cx} 3 \mathrm{Cr} 1^{\text {CreER-YFP }}-\mathrm{WT}(\mathrm{N}=4)$ and $\mathrm{C} \times 3 \mathrm{Cr} 1^{\mathrm{Cr} E \mathrm{ER}-\mathrm{YFP}}-5 \times \mathrm{XFD}(\mathrm{N}=6)$ mouse cortex stained for GFP (microglia) and Cotl1. Arrow indicates microglia immunopositive for GFP (to detect microglia) and Cotl1. B Representative images of 9-10 month old WT and 5xFAD cortex used for quantitative analysis of Msn fluorescence intensity shown in a histogram below. Unpaired t-test, ***** $p<0.0001$.

Additional file 7: Figure S5. Moesin protein levels are increased in human AD and FTLD-TDP brain. A Msn protein abundance in dorsolateral prefrontal cortex post-mortem brain tissue of control $(N=43), A D(N=$ 47), FTLD-TDP $(N=29)$, ALS $(N=54)$, and PD/PDD $(N=76)$ cases; UPENN cohort. Protein abundance was measured with LFQ [40]. One-way ANOVA, Tukey post hoc: ${ }^{*} p<0.05,{ }^{* * *} p<0.001$. B Msn protein abundance in precuneus post-mortem brain tissue of control $(N=13)$, AsymAD ( $N=$ 13), and $A D(N=20)$ cases; BLSA cohort. Protein abundance was measured with LFQ [40]. One-way ANOVA, Tukey post hoc: ${ }^{*} p<0.05,{ }^{* * *} p<$ 0.001 . C Msn protein abundance in laser capture microdissected A $\beta$ plaques from human post- mortem brain tissue of rapid progression $A D$ $(N=22)$ and sporadic $A D(N=22)$ cases. Protein abundance was measured with LFQ by Drummond et al. [42]. Unpaired t-test: ${ }^{* *} p<0.001$.

Additional file 8: Figure S6. Moesin protein and mRNA levels increase with age in $5 \times F A D$ brain. A Normalized relative Msn protein abundance in brains of wild-type (WT), 5xFAD (A $\beta$ pathology), JNPL3 (Tau pathology), and $a$ cross of $5 \times$ FAD and JNPL3 (AB \& tau pathologies) mice at 4 months, 7 months, and 10 months of age ( $N=3$ /age/genotype). Protein abundance obtained by TMT-MS [43]. B Relative Msn expression, shown as FPKM values, in brains of WT and 5xFAD mice at 3 months, 6 months, and 12 months of age ( $N=2-3 /$ age/genotype) from a previously published study [44]. Error bars represent \pm SEM. One-way ANOVA, Tukey post hoc analysis: ${ }^{*} p<0.05,{ }^{* * *} p<0.001,{ }^{* * *} p<0.0001$.

\section{Abbreviations}

Aß: Amyloid-beta; ACN: Acetonitrile; AD: Alzheimer's disease; ANOVA: Analysis of variance; AGC: Automatic gain control; AsymAD: Asymptomatic Alzheimer's disease; BCA: Bicinchoninic acid; CE: Collision energy; COBRA: Concurrent brain cell type acquisition; DAM: Disease-associated-microglia; DAPI: Diamidino-2-phenylindole; DTT: Dithiothreitol; ER: Endoplasmic reticulum; ERM: Ezrin-radixin-moesin; FDR: False discovery rate; FBS: Fetal bovine serum; FACS: Fluorescence activated cell sorting; FA: Formic acid; $f A \beta 42-488$ : fibrillar fluorescent $A \beta 42$ conjugated to HiLyte Fluor 488; GO: Gene ontology; GWAS: Genome-wide association studies; HCD: Higher energy collision dissociation; IAA: lodoacetamide; KD: Knockdown; LFQ: Label-free quantitation; LPS: Lipopolysaccharide; MACS: Magnetic-activated cell sorting; MS: Mass spectrometry; MMSE: Mini-mental status examination; Msn: Moesin; PBS: Phosphate buffered saline; PE: Phycoerythrin; PSM: Peptide spectral match; qRT-PCR: quantitative reverse transcriptase pcr; rpAD: rapidly progressive ad; RT: Room temperature; SNPs: Single-nucleotide polymorphisms; siRNA: small interfering ribonucleic acid; SAD: sporadic ad; SIP: Stock isotonic percoll; SPS: Synchronous precursor selection; TMT: Tandem mass tag; TEAB: Triethylammonium bicarbonate; TFA: Triflouroacetic acid; TBS: Tris buffered saline; WGCNA: Weighted correlation network analysis

\section{Acknowledgements}

Research reported in this publication was also supported in part by the Emory Flow Cytometry Core (EFCC) and Emory University Integrated Cellular Imaging (ICI) Microscopy Core, two of the Emory Integrated Core Facilities (EICF). EFCC is subsidized by the Emory University School of Medicine and additional support was provided by the National Center for Georgia Clinical \& Translational Science Alliance of the National Institutes of Health under Award Number UL1TR002378. ICI Microscopy core is subsidized by the Emory University Integrated Cellular Imaging Microscopy Core of the Emory Neuroscience NINDS Core Facilities grant, 5P30NS055077. The content is solely the responsibility of the authors and does not necessarily reflect the official views of the National Institute of Health.

\section{Authors' contributions}

Conceptualization: NTS, S.Rangaraju. Methodology: S. Rayaprolu, TG, HX, S. Ramesha, LDW, JS, DMD, EBD, JAW, RB. Investigation: S. Rayaprolu, NTS, S.Rangaraju. Writing-Original draft: S. Rayaprolu, NTS, S.Rangaraju. WritingReview and Editing: TG, HX, S. Ramesha, LDW, JS, DMD, EBD, JAW, JJL, LBW, RB, AIL. Funding acquisition: S. Rayaprolu, LBW, AIL, NTS, S.Rangaraju. Resources: LBW, JJL, AlL, NTS, S.Rangaraju. Supervision: NTS, S.Rangaraju. All authors read and approved the final manuscript.

\section{Funding}

Research reported in this publication was supported by the National Institute on Aging of the National Institutes of Health (Award No. F32AG064862 to S.Rayaprolu); Alzheimer's Association (Award no. 37102 to S.Rangaraju); NINDS (Award no. K08-NS099474-1 and R01 NS114130-01A1 to S.Rangaraju); additional grants by NIA (R01AG053960, R01AG057911, R01AG061800, RF1AG057471, RF1AG057470, RF1AG062181); Emory Alzheimer's Disease Research Center Grant (Award no. P50 AG025688); Accelerating Medicine Partnership for AD (U01AG046161 and U01AG061357); Additional support was provided by startup funds from George W. Woodruff School of Mechanical Engineering at the Georgia Institute of Technology (to LBW) and National Institutes of Health Cell and Tissue Engineering Biotechnology Training Grant (T32-GM008433 to LDW). 


\section{Availability of data and materials}

The mass spectrometry proteomics data have been deposited to the ProteomeXchange Consortium via the PRIDE partner repository with the dataset identifier PXD015652.

\section{Ethics approval and consent to participate}

Approval from the Emory University Institutional Animal Care and Use Committee was obtained prior to all animal-related studies (IACUC protocol \# PROTO201800252).

\section{Consent for publication}

All authors have approved of the contents of this manuscript and provided consent for publication.

\section{Competing interests}

The authors declare that they have no competing interests.

\section{Author details}

'Department of Neurology, Emory University School of Medicine, Whitehead Biomedical Research Building, 615 Michael Street, Atlanta, GA 30322, USA.

${ }^{2}$ Xiangya Hospital, Central South University, Changsha 410008, Hunan, China. ${ }^{3}$ Parker H. Petit Institute for Bioengineering and Bioscience, Wallace $\mathrm{H}$. Coulter Department of Biomedical Engineering, and Georgia W. Woodruff School of Mechanical Engineering, Georgia Institute of Technology, Atlanta, GA 30332, USA. ${ }^{4}$ Department of Biochemistry, Emory University, Atlanta, GA 30322, USA. ${ }^{5}$ School of Medicine, Emory University, Atlanta, GA 30322, USA.

\section{Received: 4 December 2019 Accepted: 24 April 2020}

\section{Published online: 07 May 2020}

\section{References}

1. Heneka MT, Carson MJ, El Khoury J, Landreth GE, Brosseron F, Feinstein DL, Jacobs AH, Wyss-Coray T, Vitorica J, Ransohoff RM, et al. Neuroinflammation in Alzheimer's disease. Lancet Neurol. 2015;14:388-405.

2. Sarlus H, Heneka MT. Microglia in Alzheimer's disease. J Clin Invest. 2017; 127:3240-9.

3. Lambert JC, Ibrahim-Verbaas CA, Harold D, Naj AC, Sims R, Bellenguez C, DeStafano AL, Bis JC, Beecham GW, Grenier-Boley B, et al. Meta-analysis of 74,046 individuals identifies 11 new susceptibility loci for Alzheimer's disease. Nat Genet. 2013;45:1452-8.

4. Efthymiou AG, Goate AM. Late onset Alzheimer's disease genetics implicates microglial pathways in disease risk. Mol Neurodegener. 2017;12:43.

5. Griffin WS, Stanley LC, Ling C, White L, MacLeod V, Perrot L, White CL 3rd, Araoz C. Brain interleukin 1 and S-100 immunoreactivity are elevated in Down syndrome and Alzheimer disease. Proc Natl Acad Sci U S A. 1989;86: 7611-5

6. Perlmutter LS, Barron E, Chui HC. Morphologic association between microglia and senile plaque amyloid in Alzheimer's disease. Neurosci Lett. 1990;119:32-6.

7. Oosterhof N, Kuil LE, van der Linde HC, Burm SM, Berdowski W, van ljcken WFJ, van Swieten JC, Hol EM, Verheijen MHG, van Ham TJ. Colonystimulating factor 1 receptor (CSF1R) regulates microglia density and distribution, but not microglia differentiation in vivo. Cell Rep. 2018;24 1203-1217 e1206.

8. Spangenberg E, Severson PL, Hohsfield LA, Crapser J, Zhang J, Burton EA, Zhang Y, Spevak W, Lin J, Phan NY, et al. Sustained microglial depletion with CSF1R inhibitor impairs parenchymal plaque development in an Alzheimer's disease model. Nat Commun. 2019;10:3758.

9. Durafourt BA, Moore CS, Zammit DA, Johnson TA, Zaguia F, Guiot MC, BarOr A, Antel JP. Comparison of polarization properties of human adult microglia and blood-derived macrophages. Glia. 2012;60:717-27.

10. Bennett ML, Bennett FC, Liddelow SA, Ajami B, Zamanian JL, Fernhoff NB, Mulinyawe SB, Bohlen CJ, Adil A, Tucker A, et al. New tools for studying microglia in the mouse and human CNS. Proc Natl Acad Sci U S A. 2016; 113:E1738-46.

11. Zhang Y, Chen K, Sloan SA, Bennett ML, Scholze AR, O'Keeffe S, Phatnani HP, Guarnieri P, Caneda C, Ruderisch N, et al. An RNA-sequencing transcriptome and splicing database of glia, neurons, and vascular cells of the cerebral cortex. J Neurosci. 2014;34:11929-47.

12. Gosselin D, Skola D, Coufal NG, Holtman IR, Schlachetzki JCM, Sajti E, Jaeger BN, O'Connor C, Fitzpatrick C, Pasillas MP, et al. An environment-dependent transcriptional network specifies human microglia identity. Science. 2017; 356:eaal3222.

13. Keren-Shaul H, Spinrad A, Weiner A, Matcovitch-Natan O, Dvir-Szternfeld R, Ulland TK, David E, Baruch K, Lara-Astaiso D, Toth B, et al. A unique microglia type associated with restricting development of Alzheimer's disease. Cell. 2017;169:1276-1290 e1217.

14. Friedman BA, Srinivasan K, Ayalon G, Meilandt WJ, Lin H, Huntley MA, Cao Y, Lee $\mathrm{SH}$, Haddick PCG, Ngu H, et al. Diverse brain myeloid expression profiles reveal distinct microglial activation states and aspects of Alzheimer's disease not evident in mouse models. Cell Rep. 2018;22:832-47.

15. Mathys H, Adaikkan C, Gao F, Young JZ, Manet E, Hemberg M, De Jager PL, Ransohoff RM, Regev A, Tsai LH. Temporal tracking of microglia activation in Neurodegeneration at single-cell resolution. Cell Rep. 2017;21:366-80.

16. Chiu IM, Morimoto ET, Goodarzi H, Liao JT, O'Keeffe S, Phatnani HP, Muratet M, Carroll MC, Levy S, Tavazoie S, et al. A neurodegeneration-specific geneexpression signature of acutely isolated microglia from an amyotrophic lateral sclerosis mouse model. Cell Rep. 2013;4:385-401.

17. Hammond TR, Dufort C, Dissing-Olesen L, Giera S, Young A, Wysoker A, Walker AJ, Gergits F, Segel M, Nemesh J, et al. Single-cell rna sequencing of microglia throughout the mouse lifespan and in the injured brain reveals complex cell-state changes. Immunity. 2019;50:253-271.e256.

18. Rangaraju S, Dammer EB, Raza SA, Rathakrishnan P, Xiao H, Gao T, Duong DM, Pennington MW, Lah JJ, Seyfried NT, Levey Al. Identification and therapeutic modulation of a pro-inflammatory subset of disease-associatedmicroglia in Alzheimer's disease. Mol Neurodegener. 2018;13:24.

19. de Sousa AR, Penalva LO, Marcotte EM, Vogel C. Global signatures of protein and mRNA expression levels. Mol BioSyst. 2009:5:1512-26.

20. Vogel C, Marcotte EM. Insights into the regulation of protein abundance from proteomic and transcriptomic analyses. Nat Rev Genet. 2012;13:22732.

21. Maier T, Guell M, Serrano L. Correlation of mRNA and protein in complex biological samples. FEBS Lett. 2009;583:3966-73.

22. Sharma K, Schmitt S, Bergner CG, Tyanova S, Kannaiyan N, Manrique-Hoyos N, Kongi K, Cantuti L, Hanisch UK, Philips MA, et al. Cell type- and brain region-resolved mouse brain proteome. Nat Neurosci. 2015;18:1819-31.

23. Flowers A, Bell-Temin H, Jalloh A, Stevens SM Jr, Bickford PC. Proteomic anaysis of aged microglia: shifts in transcription, bioenergetics, and nutrient response. J Neuroinflammation. 2017;14:96.

24. Guergues J, Zhang P, Liu B, Stevens SM Jr. Improved methodology for sensitive and rapid quantitative proteomic analysis of adult-derived mouse microglia: application to a novel in vitro mouse microglial cell model. Proteomics. 2019;19:e1800469.

25. Rangaraju S, Dammer EB, Raza SA, Gao T, Xiao H, Betarbet R, Duong DM, Webster JA, Hales CM, Lah JJ, et al. Quantitative proteomics of acutelyisolated mouse microglia identifies novel immune Alzheimer's diseaserelated proteins. Mol Neurodegener. 2018;13:34.

26. Rangaraju S, Raza SA, Li NX, Betarbet R, Dammer EB, Duong D, Lah JJ, Seyfried NT, Levey Al. Differential phagocytic properties of CD45(low) microglia and CD45(high) brain mononuclear phagocytes-activation and age-related effects. Front Immunol. 2018;9:405

27. Gao T, Jernigan J, Raza SA, Dammer EB, Xiao H, Seyfried NT, Levey Al, Rangaraju S. Transcriptional regulation of homeostatic and disease-associatedmicroglial genes by IRF1, LXRbeta, and CEBPalpha. Glia. 2019;67:1958-75.

28. Ping L, Duong DM, Yin L, Gearing M, Lah JJ, Levey Al, Seyfried NT. Global quantitative analysis of the human brain proteome in Alzheimer's and Parkinson's disease. Sci Data. 2018;5:180036.

29. Kall L, Canterbury JD, Weston J, Noble WS, MacCoss MJ. Semi-supervised learning for peptide identification from shotgun proteomics datasets. Nat Methods. 2007:4:923-5.

30. Seyfried NT, Dammer EB, Swarup V, Nandakumar D, Duong DM, Yin L, Deng Q, Nguyen T, Hales CM, Wingo T, et al. A multi-network approach identifies protein-specific co-expression in asymptomatic and symptomatic Alzheimer's disease. Cell Syst. 2017:4:60-72 e64.

31. Umoh ME, Dammer EB, Dai J, Duong DM, Lah JJ, Levey Al, Gearing M, Glass JD, Seyfried NT. A proteomic network approach across the ALS-FTD disease spectrum resolves clinical phenotypes and genetic vulnerability in human brain. EMBO Mol Med. 2018;10:48-62.

32. Dai J, Johnson ECB, Dammer EB, Duong DM, Gearing M, Lah JJ, Levey Al, Wingo TS, Seyfried NT. Effects of APOE genotype on brain proteomic network and cell type changes in Alzheimer's disease. Front Mol Neurosci. 2018;11:454. 
33. Littman DR. An inducible cre recombinase driven by Cx3cr1. MGI Direct Data Submission; 2013.

34. Liao C, Prabhu KS, Paulson RF. Monocyte-derived macrophages expand the murine stress erythropoietic niche during the recovery from anemia. Blood. 2018;132:2580-93.

35. Schindelin J, Arganda-Carreras I, Frise E, Kaynig V, Longair M, Pietzsch T, Preibisch S, Rueden C, Saalfeld S, Schmid B, et al. Fiji: an open-source platform for biological-image analysis. Nat Methods. 2012;9:676-82.

36. Gordon R, Hogan CE, Neal ML, Anantharam V, Kanthasamy AG, Kanthasamy A. A simple magnetic separation method for high-yield isolation of pure primary microglia. J Neurosci Methods. 2011;194:287-96.

37. Marek R, Caruso M, Rostami A, Grinspan JB, Das Sarma J. Magnetic cell sorting: a fast and effective method of concurrent isolation of high purity viable astrocytes and microglia from neonatal mouse brain tissue. J Neurosci Methods. 2008:175:108-18.

38. Rangaraju S, Raza SA, Pennati A, Deng Q, Dammer EB, Duong D, Pennington MW, Tansey MG, Lah JJ, Betarbet R, et al. A systems pharmacology-based approach to identify novel Kv1.3 channel-dependent mechanisms in microglial activation. J Neuroinflammation. 2017;14:128.

39. Chen MJ, Ramesha S, Weinstock LD, Gao T, Ping L, Xiao H, Dammer EB, Duong DD, Levey Al, Lah JJ, et al. Microglial ERK signaling is a critical regulator of pro-inflammatory immune responses in Alzheimer's disease. bioRxiv. 2019:798215.

40. Johnson ECB, Dammer EB, Duong DM, et al. Large-scale proteomic analysis of Alzheimer's disease brain and cerebrospinal fluid reveals early changes in energy metabolism associated with microglia and astrocyte activation. Nat Med. 2020. https://doi.org/10.1038/s41591-020-0815-6.

41. Zhang B, Gaiteri C, Bodea LG, Wang Z, McElwee J, Podtelezhnikov AA, Zhang C, Xie T, Tran L, Dobrin R, et al. Integrated systems approach identifies genetic nodes and networks in late-onset Alzheimer's disease. Cell. 2013;153:707-20.

42. Drummond E, Nayak S, Faustin A, Pires G, Hickman RA, Askenazi M, Cohen M, Haldiman T, Kim C, Han X, et al. Proteomic differences in amyloid plaques in rapidly progressive and sporadic Alzheimer's disease. Acta Neuropathol. 2017;133:933-54.

43. Kim DK, Park J, Han D, Yang J, Kim A, Woo J, Kim Y, Mook-Jung I. Molecular and functional signatures in a novel Alzheimer's disease mouse model assessed by quantitative proteomics. Mol Neurodegener. 2018;13:2.

44. Bai B, Wang X, Li Y, Chen PC, Yu K, Dey KK, Yarbro JM, Han X, Lutz BM, Rao $S$, et al. Deep multilayer brain proteomics identifies molecular networks in Alzheimer's disease progression. Neuron. 2020;105:975-991 e977.

45. Colonna M, Butovsky O. Microglia function in the central nervous system during health and Neurodegeneration. Annu Rev Immunol. 2017;35:441-68.

46. Esser J, Rakonjac M, Hofmann B, Fischer L, Provost P, Schneider G, Steinhilber D, Samuelsson B, Radmark O. Coactosin-like protein functions as a stabilizing chaperone for 5-lipoxygenase: role of tryptophan 102. Biochem J. 2009;425:265-74

47. Kim J, Shapiro MJ, Bamidele AO, Gurel P, Thapa P, Higgs HN, Hedin KE, Shapiro VS, Billadeau DD. Coactosin-like 1 antagonizes cofilin to promote lamellipodial protrusion at the immune synapse. PLoS One. 2014;9:e85090.

48. Brock TG. Capturing proteins that bind polyunsaturated fatty acids: demonstration using arachidonic acid and eicosanoids. Lipids. 2008;43:1619 .

49. Bretscher A, Edwards K, Fehon RG. ERM proteins and merlin: integrators at the cell cortex. Nat Rev Mol Cell Biol. 2002;3:586-99.

50. Berryman M, Franck Z, Bretscher A. Ezrin is concentrated in the apical microvilli of a wide variety of epithelial cells whereas moesin is found primarily in endothelial cells. J Cell Sci. 1993;105(Pt 4):1025-43.

51. Shcherbina A, Bretscher A, Kenney DM, Remold-O'Donnell E. Moesin, the major ERM protein of lymphocytes and platelets, differs from ezrin in its insensitivity to calpain. FEBS Lett. 1999;443:31-6.

52. Faure S, Salazar-Fontana LI, Semichon M, Tybulewicz VL, Bismuth G, Trautmann A, Germain RN, Delon J. ERM proteins regulate cytoskeleton relaxation promoting T cell-APC conjugation. Nat Immunol. 2004;5:272.

53. Balsis S, Benge JF, Lowe DA, Geraci L, Doody RS. How do scores on the ADAS-cog, MMSE, and CDR-SOB correspond? Clin Neuropsychol. 2015;29: 1002-9

54. Braak H, Alafuzoff I, Arzberger T, Kretzschmar H, Del Tredici K. Staging of Alzheimer disease-associated neurofibrillary pathology using paraffin sections and immunocytochemistry. Acta Neuropathol. 2006;112:389-404.
55. Hyman BT, Phelps CH, Beach TG, Bigio EH, Cairns NJ, Carrillo MC, Dickson DW, Duyckaerts C, Frosch MP, Masliah E, et al. National Institute on AgingAlzheimer's Association guidelines for the neuropathologic assessment of Alzheimer's disease. Alzheimers Dement. 2012;8:1-13.

56. Reiman EM, Chen K, Liu X, Bandy D, Yu M, Lee W, Ayutyanont N, Keppler J, Reeder SA, Langbaum JB, et al. Fibrillar amyloid-beta burden in cognitively normal people at 3 levels of genetic risk for Alzheimer's disease. Proc Natl Acad Sci U S A. 2009;106:6820-5.

57. Chen WT, Lu A, Craessaerts K, Pavie B, Frigerio CS, Mancuso R, Qian X, Lalakova J, Kühnemund M, Voytyuk I, et al. Spatial and temporal transcriptomics reveal microglia-astroglia crosstalk in the amyloid- $\beta$ plaque cell niche of Alzheimer's disease. bioRxiv. 2019:719930.

58. Masuda T, Sankowski R, Staszewski O, Bottcher C, Amann L, Sagar SC, Nessler S, Kunz P, van Loo G, et al. Spatial and temporal heterogeneity of mouse and human microglia at single-cell resolution. Nature. 2019;566:38892.

59. Bottcher C, Schlickeiser S, Sneeboer MAM, Kunkel D, Knop A, Paza E, Fidzinski P, Kraus L, Snijders GJL, Kahn RS, et al. Human microglia regional heterogeneity and phenotypes determined by multiplexed single-cell mass cytometry. Nat Neurosci. 2019;22:78-90.

60. Ting $L$, Rad R, Gygi SP, Haas W. MS3 eliminates ratio distortion in isobaric multiplexed quantitative proteomics. Nat Methods. 2011:8:937-40.

61. McAlister GC, Nusinow DP, Jedrychowski MP, Wuhr M, Huttlin EL, Erickson BK, Rad R, Haas W, Gygi SP. MultiNotch MS3 enables accurate, sensitive, and multiplexed detection of differential expression across cancer cell line proteomes. Anal Chem. 2014:86:7150-8.

62. Thompson A, Schafer J, Kuhn K, Kienle S, Schwarz J, Schmidt G, Neumann T, Johnstone R, Mohammed AK, Hamon C. Tandem mass tags: a novel quantification strategy for comparative analysis of complex protein mixtures by MS/MS. Anal Chem. 2003;75:1895-904.

63. Dammer EB, Duong DM, Diner I, Gearing M, Feng Y, Lah JJ, Levey Al, Seyfried NT. Neuron enriched nuclear proteome isolated from human brain. J Proteome Res. 2013;12:3193-206.

64. Swartzlander DB, Propson NE, Roy ER, Saito T, Saido T, Wang B, Zheng H. Concurrent cell type-specific isolation and profiling of mouse brains in inflammation and Alzheimer's disease. JCI Insight. 2018;3:121109.

65. Lankes WT, Furthmayr H. Moesin: a member of the protein 4.1-Talin-ezrin family of proteins. Proc Natl Acad Sci U S A. 1991;88:8297-301.

66. Montgomery SL, Bowers WJ. Tumor necrosis factor-alpha and the roles it plays in homeostatic and degenerative processes within the central nervous system. J Neurolmmune Pharmacol. 2012;7:42-59.

67. Michaud M, Balardy L, Moulis G, Gaudin C, Peyrot C, Vellas B, Cesari M, Nourhashemi F. Proinflammatory cytokines, aging, and age-related diseases. J Am Med Dir Assoc. 2013;14:877-82.

68. Decourt B, Lahiri DK, Sabbagh MN. Targeting tumor necrosis factor alpha for Alzheimer's disease. Curr Alzheimer Res. 2017;14:412-25.

69. Janelsins MC, Mastrangelo MA, Park KM, Sudol KL, Narrow WC, Oddo S, LaFerla FM, Callahan LM, Federoff HJ, Bowers WJ. Chronic neuron-specific tumor necrosis factor-alpha expression enhances the local inflammatory environment ultimately leading to neuronal death in 3xTg-AD mice. Am J Pathol. 2008;173:1768-82.

70. Lieberman AP, Pitha PM, Shin HS, Shin ML. Production of tumor necrosis factor and other cytokines by astrocytes stimulated with lipopolysaccharide or a neurotropic virus. Proc Natl Acad Sci U S A. 1989;86:6348-52.

71. Gong C, Qin Z, Betz AL, Liu XH, Yang GY. Cellular localization of tumor necrosis factor alpha following focal cerebral ischemia in mice. Brain Res. 1998:801:1-8.

72. Sairanen TR, Lindsberg PJ, Brenner M, Carpen O, Siren A. Differential cellular expression of tumor necrosis factor-alpha and type I tumor necrosis factor receptor after transient global forebrain ischemia. J Neurol Sci. 2001;186:8799.

73. Hashemiaghdam A, Mroczek M. Microglia heterogeneity and neurodegeneration: the emerging paradigm of the role of immunity in Alzheimer's disease. J Neuroimmunol. 2020;341:577185.

\section{Publisher's Note}

Springer Nature remains neutral with regard to jurisdictional claims in published maps and institutional affiliations. 